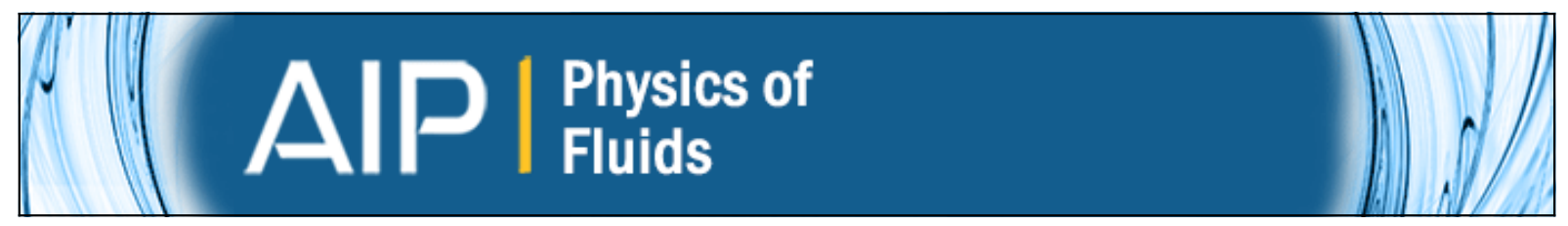

\title{
Rayleigh processes in single-phase fluids
}

M. S. Cramer

Citation: Physics of Fluids (1994-present) 18, 016101 (2006); doi: 10.1063/1.2166627

View online: http://dx.doi.org/10.1063/1.2166627

View Table of Contents: http://scitation.aip.org/content/aip/journal/pof2/18/1 ?ver=pdfcov

Published by the AIP Publishing

\section{Articles you may be interested in}

Admissible shock waves and shock-induced phase transitions in a van der Waals fluid Phys. Fluids 23, 086101 (2011); 10.1063/1.3622772

Composition dependence and the nature of endothermic freezing and exothermic melting

J. Chem. Phys. 126, 124506 (2007); 10.1063/1.2711179

Heat capacity of tetrahydrofuran clathrate hydrate and of its components, and the clathrate formation from supercooled melt

J. Chem. Phys. 124, 154507 (2006); 10.1063/1.2188944

Film cooling effectiveness on a large angle blunt cone flying at hypersonic speed

Phys. Fluids 17, 036102 (2005); 10.1063/1.1862261

Determination of equilibrium pitch of cholesteric phases by isobaric-isothermal Monte Carlo simulation J. Chem. Phys. 114, 8210 (2001); 10.1063/1.1365086

\section{AlP Re-register for Table of Content Alerts}




\title{
Rayleigh processes in single-phase fluids
}

\author{
M. S. Cramer \\ Department of Engineering Science and Mechanics, Virginia Polytechnic Institute and State University, \\ Blacksburg, Virginia 24061
}

(Received 27 July 2005; accepted 1 December 2005; published online 26 January 2006)

\begin{abstract}
In the present study we examine Rayleigh flows of single-phase, nonreacting fluids. When the fluid is of the Bethe-Zel'dovich-Thompson type, it is shown that as many as three sonic points and extrema of the entropy, enthalpy, and heat supply can occur on a fixed Rayleigh line. Exact solutions for van der Waals gases are provided, as is a complete theory of shocked and unshocked flows subjected to strict heating or strict cooling. Further nonclassical results of interest include the occurrence of flow choking in unshocked and shocked strictly cooled flows, the occurrence of as many as three shock waves in cooled or heated flows, and shock splitting in both cooled and heated flows. (C) 2006 American Institute of Physics. [DOI: 10.1063/1.2166627]
\end{abstract}

\section{INTRODUCTION}

One of the simplest models of a diabatic flow is the Rayleigh process, defined as a flow that is one dimensional, steady, and frictionless with either heat addition or extraction. Rayleigh processes are not only used to model ordinary heat transfer, but are also employed to model flows with condensation or combustion. Most discussions of Rayleigh flows are based on the perfect gas theory. However, many applications involve pressures that are so large that the ideal gas law is no longer valid. Unfortunately, there are very few studies that describe Rayleigh flows of imperfect gases. One of the most recent investigations involving nonideal gases is due to Schnerr and Leidner, ${ }^{1}$ who examined both constant and variable area diabatic flows of van der Waals gases. These authors showed a number of qualitative differences with the conventional perfect gas theory, including the possibility of a decreasing (increasing) Mach number during heat addition to a subsonic (supersonic) flow. The results of Schnerr and Leidner are seen to be consistent with the report of Cramer, ${ }^{2}$ who gave a brief description of Rayleigh and other processes in general pressurized gases. Our main objective in the present paper is to provide a more complete theory of Rayleigh processes, which describes the full range of pressures and temperatures attainable by arbitrary singlephase, nonreacting fluids.

The aforementioned studies, as well as the related work by Chandrasekar and Prasad, ${ }^{3}$ Cramer and Best, ${ }^{4}$ Cramer and Fry, ${ }^{5}$ Kluwick, ${ }^{6}$ and Cramer, Monaco, and Fabeny ${ }^{7}$ on isentropic, nozzle, and Fanno flows, strongly suggest that the most striking qualitative and quantitative differences between low- and high-pressure flows will occur for BetheZel'dovich-Thompson (BZT) fluids. The latter are ordinary substances for which the fundamental derivative of gasdynamics,

$$
\Gamma \equiv \frac{a}{\rho}+\left.\frac{\partial a}{\partial \rho}\right|_{s}=\left.\frac{V^{4}}{2 a} \frac{\partial^{2} p}{\partial V^{2}}\right|_{s},
$$

is negative over a nonzero range of pressures and temperatures corresponding to the single-phase regime. Here $\rho, s$,
$V \equiv \rho^{-1}$, and $p$ are the fluid density, entropy, specific volume, and pressure, respectively. The quantity

$$
a \equiv\left(\left.\frac{\partial p}{\partial \rho}\right|_{s}\right)^{1 / 2}=\left(-\left.V^{2} \frac{\partial p}{\partial V}\right|_{s}\right)^{1 / 2}
$$

is the thermodynamic sound speed of the fluid. The first form of (1) can be related to the second through straightforward thermodynamic manipulations. As pointed out by Bethe, ${ }^{8}$ Zel'dovich, ${ }^{9}$ Lambrakis and Thompson, ${ }^{10}$ and Thompson and Lambrakis, ${ }^{11}$ (1) will become negative in fluids having moderately large specific heats. The typical range of pressures and temperatures for which $\Gamma<0$ is depicted in Fig. 1, where lines of constant $\hat{\Gamma} \equiv \rho \Gamma / a$ have been plotted on a $p-V$ diagram for a van der Waals gas having a specific heat at a constant volume equal to $50 R$, where $R$ is the gas constant. There it is seen that $\Gamma<0$ for pressures and temperatures on the general order of the critical values. The work of Bethe, Zel'dovich, and Thompson suggests that the shape of this region of negative $\Gamma$ is typical of more general gas models. The overall size of the region will depend on the specific fluid considered and the specific equation of the state and heat capacity model used to compute its behavior. In the remainder of this paper, the term Bethe-Zel'dovichThompson (or BZT) fluid will be used to refer to any fluid having a $\Gamma<0$ region similar to that depicted in Fig. 1.

The general scope and assumptions to be applied in this study are delineated in the next section. The basic theory of Rayleigh flows in BZT fluids is developed in Sec. III. Exact solutions for Rayleigh flows of van der Waals gases are provided in Sec. IV. In Sec. V, we provide a complete description of the flow configurations possible for strictly heated and strictly cooled flows. Both shocked and unshocked flows are considered.

\section{FORMULATION}

In this paper we consider only single-phase and nonreacting fluids that are undergoing a Rayleigh process. The Rayleigh process is defined as a steady, frictionless flow having stream tubes of constant area with heat addition or sub- 


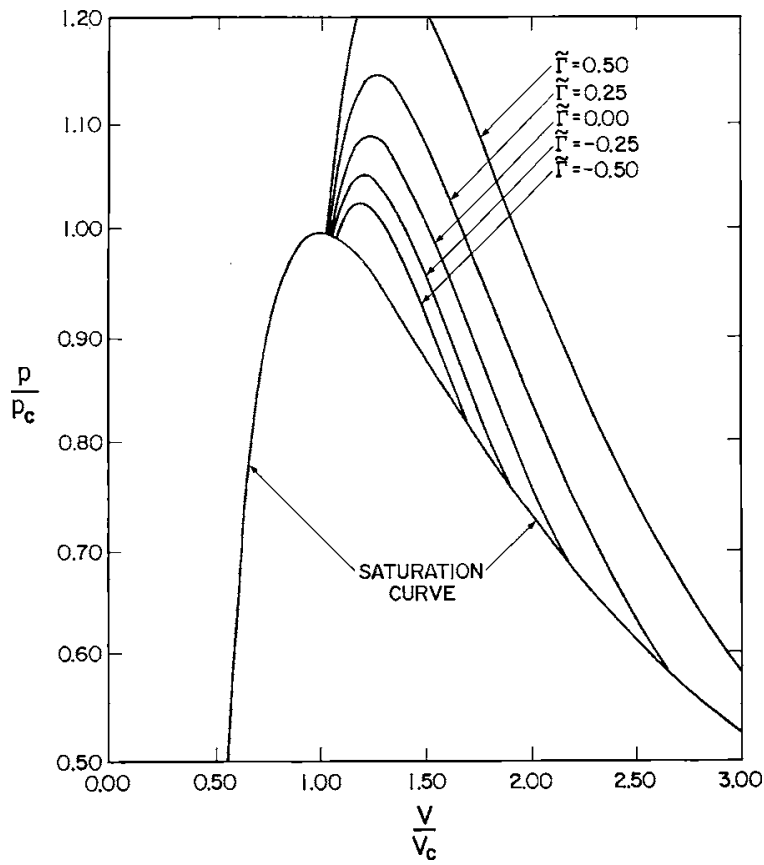

FIG. 1. Constant $\widetilde{\Gamma} \equiv \Gamma \rho / a$ contours. The fluid model is that of a van der Waals gas with $c_{v}=50 R$. The subscripts $c$ denote quantities evaluated at the thermodynamic critical point.

traction. Because heat conduction is not considered explicitly, the only inequalities constraining this flow are due to the condition of a stable thermodynamic equilibrium, i.e.,

$$
\begin{aligned}
& \left.c_{v} \equiv T \frac{\partial s}{\partial T}\right|_{\rho}>0, \\
& \left.\frac{\partial p}{\partial \rho}\right|_{T}=-\left.V^{2} \frac{\partial p}{\partial V}\right|_{T}>0,
\end{aligned}
$$

where $c_{v}=c_{v}(\rho, T)$ is the specific heat at constant volume and $T$ is the absolute temperature. Standard thermodynamic manipulations can be used to show that (3) and (4) imply

$$
\begin{aligned}
& c_{p}>c_{v}>0, \\
& \left.\frac{\partial p}{\partial V}\right|_{s}=\left.\gamma \frac{\partial p}{\partial V}\right|_{T}<0,
\end{aligned}
$$

for all $p$ and $T$ in the single-phase regime. Here

$$
c_{p}=\left.c_{p}(\rho, T) \equiv T \frac{\partial s}{\partial T}\right|_{p}
$$

is the specific heat at constant pressure and $\gamma \equiv c_{p} / c_{v}$ is the ratio of specific heats. Equation (6) is the basis for the wellknown result that both the isotherms and isentropes of a single-phase fluid have negative slopes in the $p-V$ plane.

According to (1), fluids having $\Gamma>0$ will have isentropes that are concave up in a $p-V$ plane; one such isentrope is sketched in Fig. 2(a). An isentrope that passes through a $\Gamma<0$ region similar to that plotted in Fig. 1 will have two inflection points; one such isentrope is sketched in Fig. 2(b).
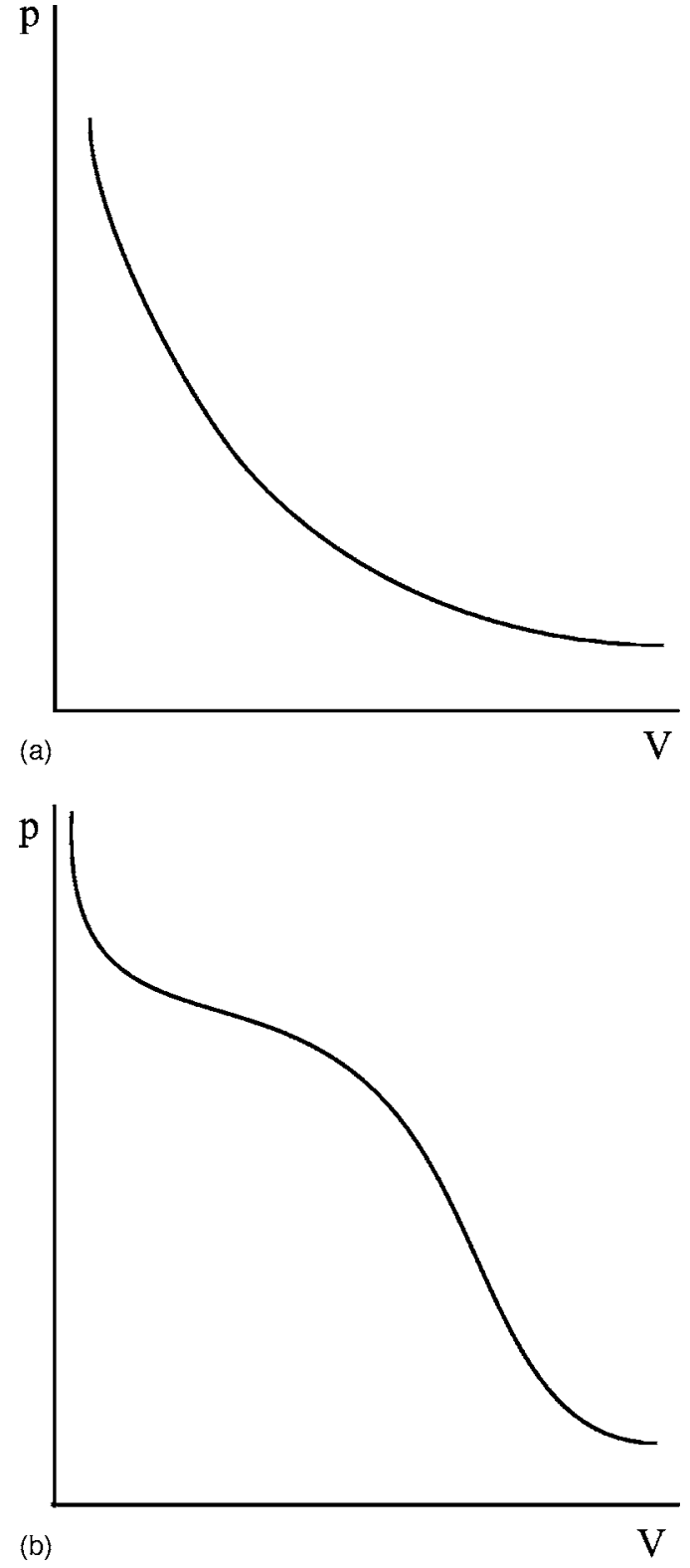

FIG. 2. Sketches of typical isentropes. (a) $\Gamma>0$; (b) isentropes passing through a $\Gamma<0$ region.

It will be shown that it is the nonconvex character of the isentropes of a BZT fluid that gives rise to most of the nonclassical behavior described here.

We will also confine our attention to fluids having $\beta>0$, where

$$
\beta \equiv-\left.\frac{1}{\rho} \frac{\partial \rho}{\partial T}\right|_{p}
$$

is referred to as the coefficient of thermal expansivity. There is no general physical principle that requires that $\beta>0$. In fact, it is well known that $\beta<0$ for liquid water at temperatures below $4{ }^{\circ} \mathrm{C}$. Nevertheless, $\beta>0$ for a great number of substances at the pressures and temperatures of interest here and we will regard $\beta>0$ for all flows discussed in the following sections.

The equations governing the Rayleigh process will be taken to be 


$$
\begin{aligned}
& \dot{m}=\rho v=\frac{v}{V}=\mathrm{const}, \\
& p+\dot{m}^{2} V=\mathrm{const},
\end{aligned}
$$

where $v$ is the material speed and $\dot{m}$ is the mass flux. Here we note that (9) is the mass equation and (10) is the principle of conservation of momentum combined with (9) for a constant area frictionless flow. Heat addition or extraction is taken into account by the following energy equation:

$$
h+\frac{\dot{m}^{2}}{2} V^{2}=Q,
$$

where $h$ is the fluid enthalpy and the mass equation has been used to replace $v$ by $\dot{m} V$. If $Q$ increases (decreases) in the flow direction, the fluid is said to be heated (cooled). An inspection of (10) reveals the well-known fact that the Rayleigh process corresponds to a straight line having a slope $-\dot{m}^{2}$ in the $p$ - $V$ plane. We will refer to this line as a Rayleigh line.

In the present paper we will also consider shocked flows. The jumps in the flow properties satisfy the usual RankineHugoniot conditions that can be written as

$$
\begin{aligned}
& \llbracket v \rho \rrbracket=0, \\
& \llbracket p+\dot{m}^{2} V \rrbracket=0, \\
& \llbracket h+\frac{\dot{m}^{2} V^{2}}{2} \rrbracket=0,
\end{aligned}
$$

where the double brackets denote jumps in the indicated quantity across the shock. If we eliminate $\dot{m}^{2}$ between (13) and (14), we obtain

$$
\llbracket h \rrbracket=\llbracket p \rrbracket \frac{V_{2}+V_{1}}{2},
$$

where the subscripts 1 and 2 denote states before and after the shock, respectively. For a given upstream thermodynamic state (15) provides the locus of downstream thermodynamic states that satisfy the mass, momentum, and energy equations. The set of states satisfying (15) is referred to as the shock adiabat or Hugoniot. The shape of a shock adiabat can be deduced by the consideration of Bethe's ${ }^{8}$ relation for the entropy jump across weak shock waves:

$$
\llbracket s \rrbracket \approx-\frac{\Gamma_{1} a_{1}}{6 T_{1} V_{1}}\left(\frac{\llbracket V \rrbracket}{V_{1}}\right)^{3}+O\left(\frac{\llbracket V \rrbracket}{V_{1}}\right)^{4},
$$

where the subscript 1 denotes properties just ahead of the shock. Relation (16) is obtained by a straightforward expansion of (15) and is the basis for the well-known result that the entropy rise across a shock is third order in the shock strength, at least if $\Gamma_{1} \rho_{1} / a_{1}=O(1)$. From (16) it may be shown that the slope and curvature of the Hugoniot is identical to that of an isentrope at the same point in a $p-V$ diagram. Thus, the shock adiabats will be concave up (down) at any point where $\Gamma>0(<0)$ in the $p$ - $V$ plane. That is, shock adiabats will have the same general shape in the $p-V$ plane as the isentropes. A sketch of a shock adiabat that passes

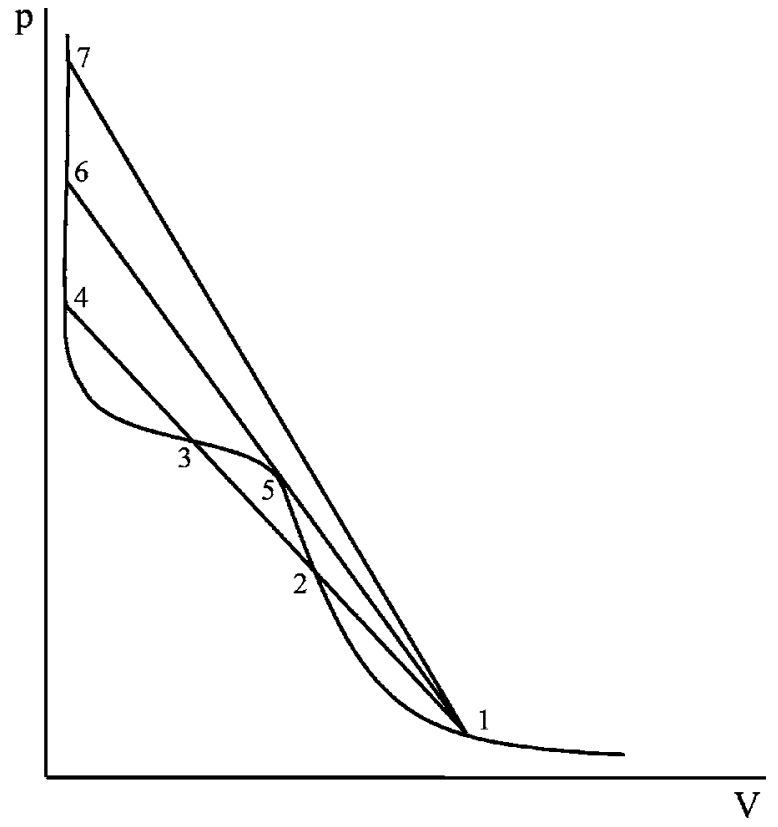

FIG. 3. Shock adiabat and sample Rayleigh lines.

through the region of $\Gamma<0$ depicted in Fig. 1 has been provided in Fig. 3.

As in the perfect gas theory, the pressure on a shock adiabat for a general fluid becomes singular at some value of specific volume. If the shock adiabat passes through the point $V_{1}, p_{1}$, the singularity occurs when

$$
1+\frac{\beta a^{2}}{2 c_{p}} \frac{V-V_{1}}{V}=0 .
$$

The portion of the shock adiabat that will be nonsingular will therefore correspond to

$$
1+\frac{\beta a^{2}}{2 c_{p}} \frac{V-V_{1}}{V}>0,
$$

which will be assumed to be satisfied throughout this study.

A useful interpretation of the shock adiabat can be obtained by considering a Rayleigh process between two points 1 and 2 . It can then be shown that (11) can be rearranged to yield

$$
h_{2}-h_{1}+\frac{\dot{m}^{2}}{2}\left(V_{2}^{2}-V_{1}^{2}\right)=Q_{2}-Q_{1} \text {. }
$$

A comparison of (9), (10), (12)-(14), and (18) reveals that shock adiabats can be regarded as a $Q=$ const curves in, say, the $p-V$ plane.

Relationships among different shock adiabats can now be obtained by noting that (10) and (11) can be combined to yield $Q=Q\left(p, V ; V_{*}, p_{*}\right)$, where the subscript * denotes an arbitrary reference point. If we hold $V_{*}, p_{*}, V$ constant and differentiate $Q$ with respect to $p$, we find that the rate of change of $Q$ along a constant $V$ line in the $p-V$ plane is given by 


$$
\left.\frac{\partial Q}{\partial p}\right|_{V}=\frac{c_{p} V}{\beta a^{2}}\left(1+\frac{\beta a^{2}}{c_{p}} \frac{V-V_{*}}{2 V}\right) .
$$

If (19) is combined with (17) it can be shown that shock adiabats corresponding to different values of $Q$ do not cross in the $p-V$ plane. Furthermore, shock adiabats corresponding to larger values of $Q$ will lie entirely above those associated with smaller values of $Q$ in the $p-V$ plane.

At a fixed upstream state, say $V_{1}, p_{1}$, Eq. (13) requires that the downstream state lies on a Rayleigh line, i.e., a straight line having a slope of $-\dot{m}^{2}$ in the $p-V$ plane. Thus, possible shock states are intersections of the Rayleigh line and a shock adiabat that passes through a given upstream state. In Fig. 3 we have sketched three Rayleigh lines and a shock adiabat that originate at a single point. It should be clear that, when the shock adiabat passes through the region of negative $\Gamma$, the Rankine-Hugoniot jump conditions (12)-(14) can have as many as four solutions.

Not all solutions to (12)-(14) correspond to physically realizable shock waves. We follow Cramer ${ }^{2,12,13}$ and employ the following Lax-Oleinik admissibility condition. ${ }^{14,15}$ Here we consider shock adiabats and Rayleigh lines in the $p$-V plane and refer to shocks across that the pressure increases (decreases) as compression (expansion) shocks. The admissibility condition can be now stated as follows: A discontinuity satisfying (12)-(14) is an admissible compression (expansion) shock iff the Rayleigh line between the proposed upstream and downstream states lies entirely above (below) the shock adiabat between those points. It can be shown ${ }^{12,13,16}$ that discontinuities satisfying this admissibility condition have a physically realizable viscous structure, result in an increase in entropy, and correspond to a speedordering relation of the form

$$
M_{1} \geqslant 1 \geqslant M_{2},
$$

where $M \equiv v / a$ is the flow Mach number and, in (20), the subscripts 1 and 2 refer to the upstream and downstream states, respectively.

As an example of the application of the above admissibility condition, we note that discontinuities represented by 1-4, 1-3, 2-4 in Fig. 3 are inadmissible because the Rayleigh line does not lie entirely above or below the adiabat between the proposed end states. On the other hand, compression shocks represented by 1-2, 3-4, and 1-7, and the expansion shock represented by 3-2 are admissible. The compression shock represented by 1-5 and 5-6 are two of many admissible shocks for which the Rayleigh line is tangent to the adiabat. Such tangency points correspond to sonic conditions, e.g., in the cases sketched in Fig. 3, $M_{5}=1$, and will be referred to as sonic shocks.

\section{GENERAL THEORY}

As pointed out in the Introduction, we will focus our attention on BZT fluids, i.e., those fluids for which $\Gamma<0$ in a region similar to that depicted in Fig. 1. In Fig. 4 we have sketched a Rayleigh line that passes through the $\Gamma<0$ region of a BZT fluid along with a few isentropes. We note that there are only two possible arrangements of the isentropes
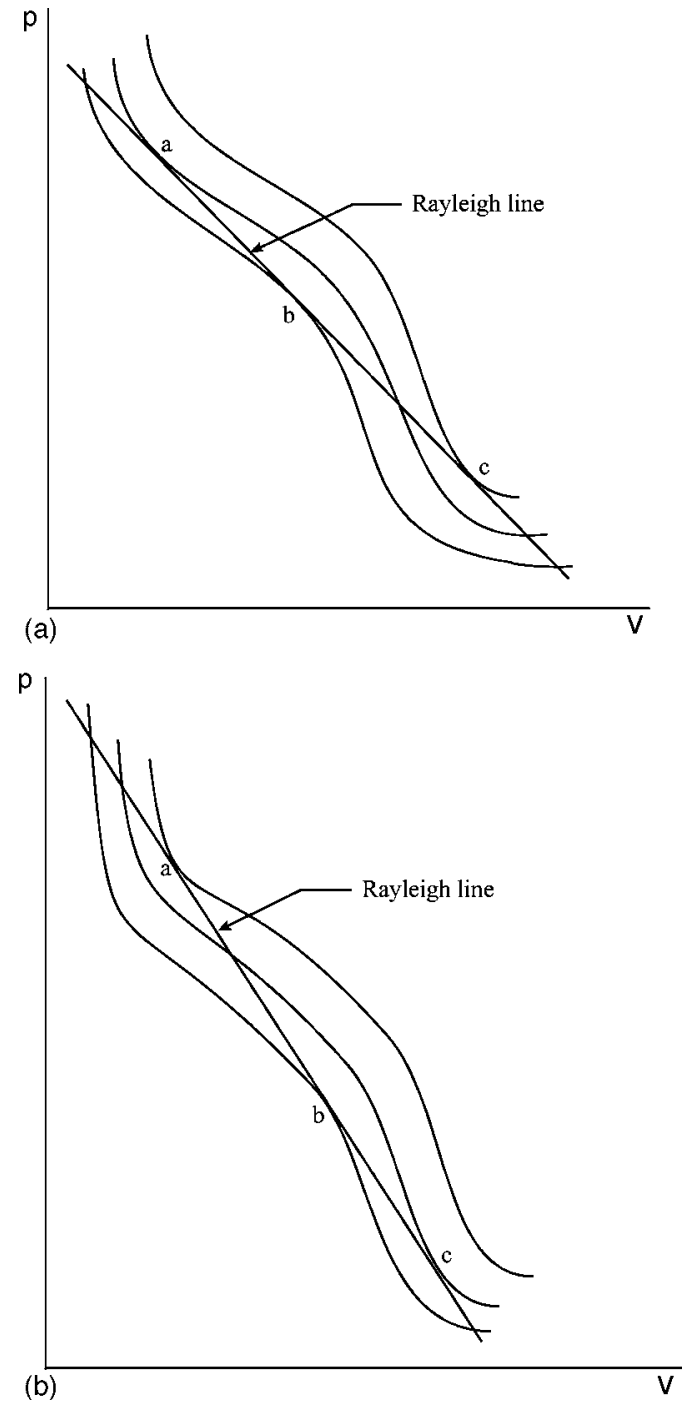

FIG. 4. Rayleigh line and isentropes that pass through the $\Gamma<0$ region depicted in Fig. 1. (a) $s_{c}>s_{a}>s_{b}$; (b) $s_{a}>s_{c}>s_{b}$.

and the Rayleigh line; these are depicted in Figs. 4(a) and 4(b). Standard thermodynamic manipulations combined with (3) and (4) and the condition that $\beta>0$ can be used to show that

$$
\left.\frac{\partial s}{\partial p}\right|_{V}=\frac{c_{p} V}{T \beta a^{2}}>0
$$

As a result, isentropes do not cross and isentropes corresponding to larger pressures carry larger values of entropy. We may immediately conclude that $s_{c}>s_{a}>s_{b}$ for the case depicted in Fig. 4(a) and $s_{a}>s_{c}>s_{b}$ in the case depicted in Fig. 4(b). Inspection of the isentropes that are nowhere tangent to the Rayleigh line reveals that points $a$ and $c$ constitute local maxima of the entropy variation along the Rayleigh line and point $b$ represents a local minimum in the entropy as it varies along the Rayleigh line. As a result, the entropy variation along the Rayleigh line must necessarily be that sketched in Fig. 5. The existence of three extrema of entropy contrasts sharply with the case of a perfect gas in which the entropy variation along a Rayleigh line only has a single local maximum. We note that the reasoning given 


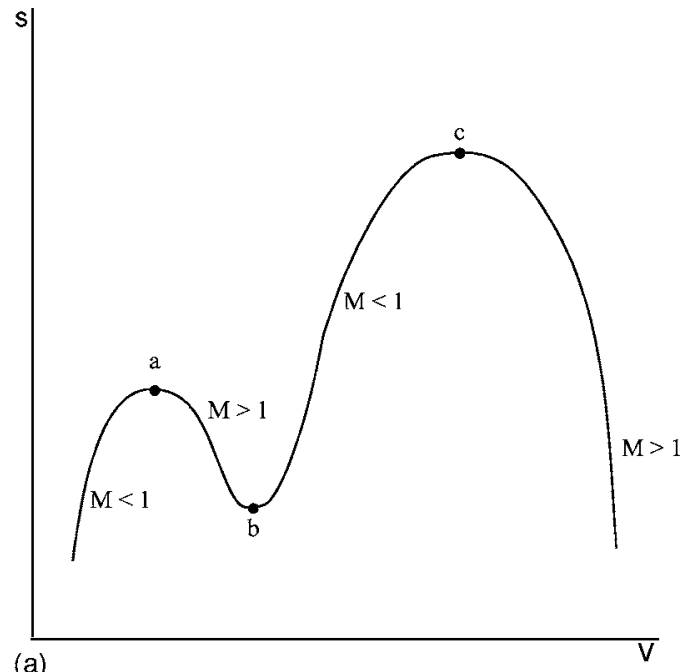

(a)

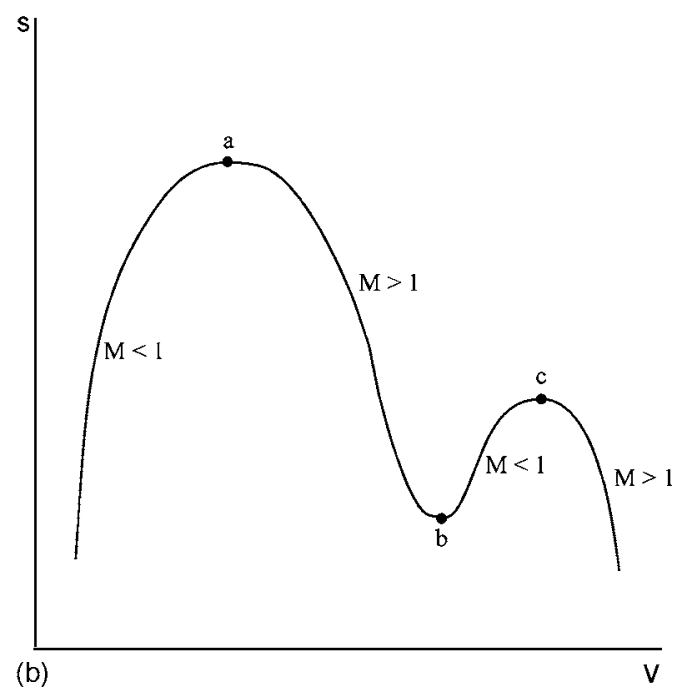

FIG. 5. Variation of entropy and Mach number along Rayleigh lines. (a) $s_{c}>s_{a}>s_{b} ;$ (b) $s_{a}>s_{c}>s_{b}$.

above can be applied to the perfect gas case once it is recognized that

$$
\hat{\Gamma}=\frac{\gamma+1}{2}>1
$$

for a perfect gas and therefore that the isentropes are necessarily concave up.

Insight into the Mach number variation along a Rayleigh line associated with Figs. 4 and 5 can be deduced by differentiating (10) to yield

$$
\left.\frac{d p}{d V}\right|_{R}=-\dot{m}^{2}=-\frac{M^{2} a^{2}}{V^{2}}
$$

where the subscript $R$ denotes a quantity evaluated on the Rayleigh line or a derivative taken along the Rayleigh line and $M \equiv v / a$ is the Mach number. If we combine (2) with (23), it is easily shown that

$$
\left.\frac{\partial p}{\partial V}\right|_{s}-\left.\frac{d p}{d V}\right|_{R}=\frac{a^{2}}{V^{2}}\left(M^{2}-1\right) .
$$

Thus,

$$
M \lessgtr 1 \text { iff }\left.\left.\frac{\partial p}{\partial V}\right|_{s} \lessgtr \frac{d p}{d V}\right|_{R},
$$

and we can deduce whether the flow is supersonic or subsonic simply by comparing the slopes of the isentropes and the Rayleigh line at their intercepts. Furthermore, it should be clear that a tangency point corresponds to both an extremum of $s$ and a sonic condition and that any Rayleigh line passing through a region of $\Gamma<0$, similar to that sketched in Fig. 1, necessarily has three sonic points. We have marked the regions of supersonic and subsonic flow in Fig. 5.

The existence of three sonic points on the Rayleigh line not only contrasts with the well known theory of perfect gases but also requires that the Mach number variation with $V$ or $\rho$ is nonmonotone. As a result, the commonly employed practice of expressing the variations of thermodynamic properties in terms of the Mach number is not appropriate for BZT fluids.

A direct relation between the entropy variation and the Mach number can be derived by regarding $p=p(V, s)$ in (23) to obtain

$$
\left.\frac{\partial p}{\partial V}\right|_{s}+\left.\left.\frac{d s}{d V}\right|_{R} \frac{\partial p}{\partial s}\right|_{V}=-\frac{M^{2} a^{2}}{V^{2}} .
$$

If we use (2) and the inverse of (21), we find that

$$
\left.T \frac{d s}{d V}\right|_{R}=\frac{a^{2}}{V} \frac{1-M^{2}}{G},
$$

where

$$
G \equiv \frac{\beta a^{2}}{c_{p}}=\frac{\gamma-1}{\beta T}=\left.\frac{1}{\rho c_{v}} \frac{\partial p}{\partial T}\right|_{\rho}>0
$$

is the Grüneisen parameter discussed by Arp et al. ${ }^{17}$ in the context of Fanno, Rayleigh, and isentropic flows. Each form of (27) can be related to the others by straightforward thermodynamic manipulations. Equation (26) can be shown to be completely consistent with our conclusions based on Fig. 4 and, when combined with (24), provides relationships among the entropy variation, the Mach number, and the relative slopes of the Rayleigh line and the isentropes.

The variation of the Mach number along a Rayleigh line can be related to the fundamental derivative (1) by the following equation:

$$
\left.\frac{d M}{d \rho}\right|_{R}=-\left.V^{2} \frac{d M}{d V}\right|_{R}=-\frac{M}{\rho}\left(\hat{\Gamma}+\left.\frac{a}{G T} \frac{\partial a}{\partial s}\right|_{\rho}\left(M^{2}-1\right)\right) .
$$

Equation (28) was derived by combining the differential of the mass, Eq. (9), the definitions of the Mach number and the fundamental derivative (1) and (26). Analogous results were obtained by Cramer and Best ${ }^{4}$ for isentropic flows and by Cramer, Monaco, and Fabeny ${ }^{7}$ for Fanno flows. From (28) we can immediately conclude that the slope of the $M$ vs $V$ curve is positive (negative) at a sonic point iff $\Gamma$ is positive (negative) at that point.

If $M \neq 1$, then the sign of the right-hand side of (28) will depend on the sign of 


$$
\left.\frac{\partial a}{\partial s}\right|_{\rho}
$$

Although this derivative is positive in the ideal gas limit, no general conclusions can be made about its sign for general single-phase fluids.

In Fig. 5 points $a$ and $c$ were seen to correspond to local maxima and the point $b$ was seen to correspond to a local minimum in the entropy. We may relate the type of extremum to (1) by combining (28) with the derivative of (26) to obtain

$$
\left.\frac{d^{2} s}{d V^{2}}\right|_{R, M=1}=-\left(\frac{2 a^{2}}{V^{2} G T} \hat{\Gamma}\right)_{M=1},
$$

which verifies that $s$ is a local maximum if the sonic point occurs in the $\Gamma>0$ region and is a local minimum if the sonic point occurs at a $\Gamma<0$ point.

We now consider the variation of the enthalpy $h \equiv e$ $+p V$ along the Rayleigh line. Here $e \equiv e(V, T)$ is the internal energy. This variation can be determined by combining Gibbs' relation

$$
d h=T d s+V d p,
$$

with (23) and (26) to obtain

$$
\left.\frac{d h}{d V}\right|_{R}=\frac{a^{2}}{V G}\left[1-M^{2}(1+G)\right] .
$$

As in the case of the perfect gas theory, the extrema of $h$ occur at subsonic, rather than sonic, points. In the perfect gas limit $G \rightarrow \gamma-1$, so that the slope of the $h-V$ curve vanishes when $M=\gamma^{-1 / 2}$ (=const). Because the Mach number increases monotonically with $V$ for a perfect gas, $h-V$ or $h-s$ curves will have one and only one local maximum in $h$, provided the Rayleigh process starts at a sufficiently low Mach number. For general fluids $G \neq$ const and, for at least BZT fluids, $M$ is not necessarily a monotone function of $V$ on a Rayleigh line. The variation of $h$ is therefore expected to be more complex than in the perfect gas theory. Cramer $^{2}$ has provided a brief discussion of the enthalpy variation that was based on the assumption of constant $G$. Below we provide a discussion based on somewhat weaker restrictions.

Before developing the Mollier diagrams, i.e., the $h-s$ curves for BZT fluids, we present two more results of general interest. The first is the required variation of the heat supply $Q$. If we differentiate (11) with respect to $V$ and use (23) and (30), we find

$$
\left.\frac{d Q}{d V}\right|_{R}=\left.T \frac{d s}{d V}\right|_{R}
$$

Thus, the $Q$ vs $V$ curve will have the same qualitative appearance as the $s$ vs $V$ curves seen in Fig. 5. In particular, subsonic heating of the flow will drive the flow toward one of the two maxima. If the initial state is between points $a$ and $c$ in Fig. 5, heating will drive the flow away from the local minimum in $s$ at $b$ and cooling will ultimately lead to choking at the sonic point $b$.

Conditions (26), (28), and (29) require that $\Gamma<0$ and

$$
\left.\frac{d M}{d V}\right|_{R}<0
$$

at the sonic point $b$ in Fig. 5. Thus, if we consider a subsonic point near $b$, then heating the flow can give rise to a decrease, rather than the classical increase, in the Mach number. In like manner, cooling a supersonic flow near $b$ can result in a decrease in the Mach number. Thus, the more general analysis presented here is seen to be consistent with the Schnerr and Leidner ${ }^{1}$ discussion of van der Waals gases.

The second result is that for the temperature variation. If we take $s=s(T, p)$ in (26) and use (7) and the fact that

$$
\left.\frac{\partial s}{\partial p}\right|_{T}=-\beta V,
$$

we find that

$$
\left.\frac{d T}{d V}\right|_{R}=\frac{1-\gamma M^{2}}{\beta V} .
$$

Thus, the temperature has a local maximum or minimum when $M=\gamma^{-1 / 2}<1$. Although $\gamma \neq$ const for a general fluid undergoing a Rayleigh process, the Mach number at which the temperature becomes a maximum or a minimum has the same expression, i.e., $\gamma^{-1 / 2}$, as that for a perfect gas.

In the following we present a discussion of the Mollier diagram corresponding to a BZT fluid. To provide definite conclusions, we will need to make certain simplifying assumptions on the variation of $G$ and $M$. We will therefore require that $G$ decreases monotonically with increasing $V$ and that the Mach number has no more than one local maximum and one local minimum. We will allow for this nonmonotone variation of $M$ even if only one sonic point occurs on the Rayleigh line. Although each of these assumptions is made simply for convenience, they are consistent with the specific numerical solutions described in Sec. IV and reasoning based on van der Waals gases.

We first consider the $M$ and $(1+G)^{1 / 2}$ variation sketched in Fig. 6(a). This case contains three sonic points, denoted by $a, b$, and $c$ and a single intersection of the $M$ vs $V$ curve and the $(1+G)^{-1 / 2}$ vs $V$ curve at point $d$. If we employ (26) and (31), we find that the corresponding $h$-s curve is that sketched in Fig. 6(b). We note that this case could also give rise to a $h$-s diagram that is identical to Fig. 6(b), except that $s_{c}>s_{a}$.

As pointed out in the discussion of Figs. 4 and 5, the three sonic points correspond to two maxima of $s$ (at $a$ and $c$ in Fig. 6) and a minimum of $s$ at the point $b$ seen in Fig. 6. The upper lobe of the $h-s$ curve is qualitatively similar to that of the perfect gas theory. On the other hand, the lower lobe, i.e., the portion of the $h-s$ curve in the vicinity of point $c$, does not have a local maximum in $h$. The associated temperature distribution could also be deduced through the use of (33). If the initial Mach number is taken to be sufficiently small, the temperature will have a local maximum at some point to the left of point (a) in Fig. 6. There may or may not be a local minimum and a local maximum in $T$ in the neighborhood of points $b$ and $c$, respectively. 

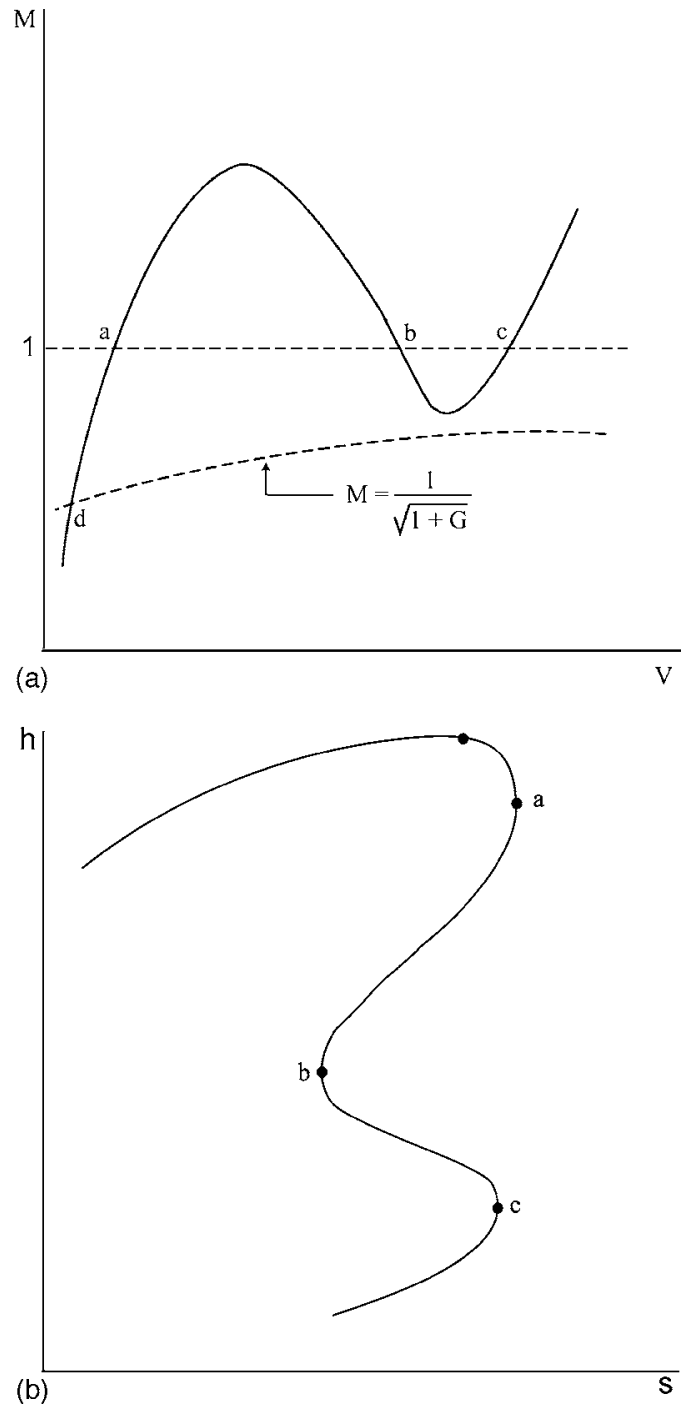

FIG. 6. $M-V$ and $h$-s diagrams. Example of a flow having three sonic points and one extremum of $h$.

We next consider the $M$ and $(1+G)^{-1 / 2}$ variation sketched in Fig. 7(a). In this case there are three points at which $M=(1+G)^{-1 / 2}$. As a result, the primary difference between the $h-s$ curves in Fig. 6(a) and Fig. 7(b) will be the existence of three, rather than one, extrema of $h$. In this case, the $h$-s diagram in the neighborhood of the sonic points $a$ and $c$ in Fig. 7 is qualitatively the same as that of the classical perfect gas case. The curve in the $\Gamma<0$ region is also similar to the perfect gas theory, except that both $s$ and $h$ attain local minima rather than maxima.

We note that Fig. 7(b) has been sketched, using the assumption that $s_{c}>s_{a}$. It is also possible that $s_{a}>s_{c}$ for this case. We have made no assumptions on the behavior of the ratio of specific heats, $\gamma$, on the Rayleigh line so that no general conclusions can be made regarding the number of extrema of the temperature. However, if $\gamma$ varies monotonically with $V$ and is always less than $G+1$, then the case depicted in Fig. 7 will always give rise to a local minimum in temperature between points $b$ and $e$ and local maxima between points $d$ and $a$ and points $f$ and $c$.

The last nonclassical case to be considered is depicted in
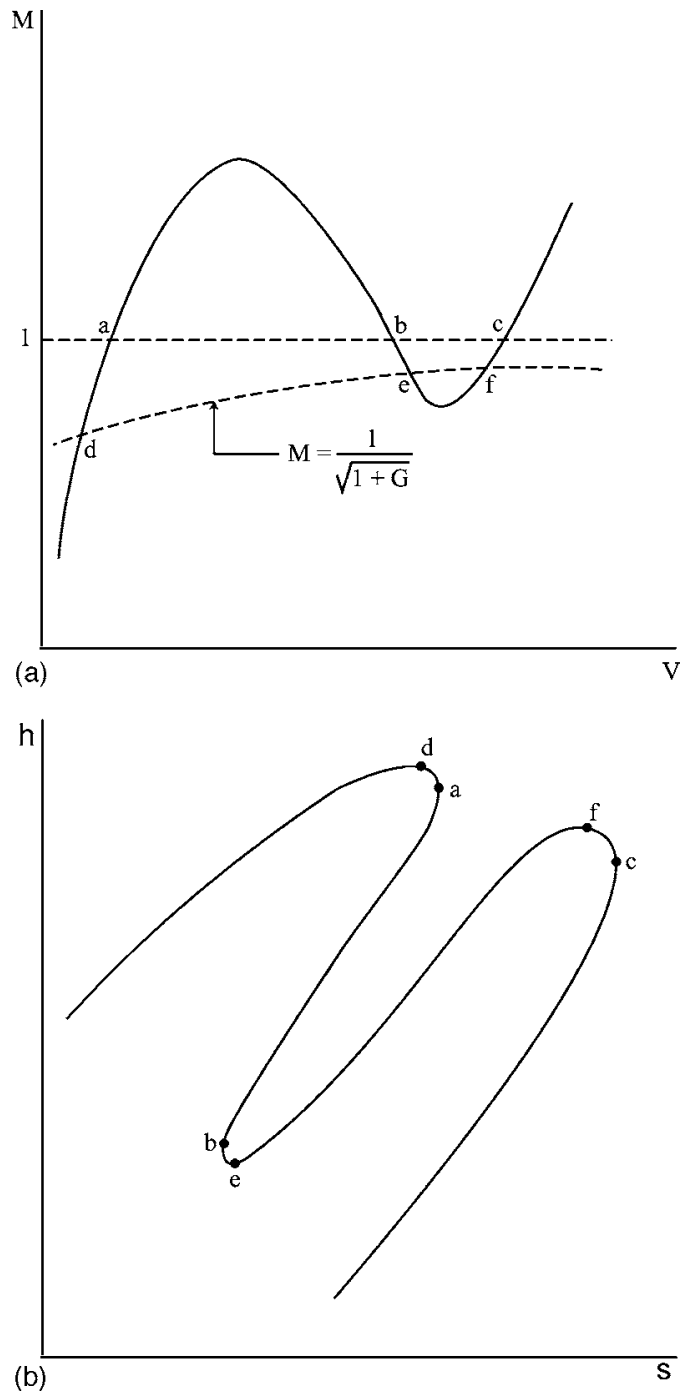

FIG. 7. $M-V$ and $h$-s diagrams. Example of a flow having three sonic points and three extrema of $h$.

Fig. 8. In this case, only one sonic point occurs but there are three points at which $M=(1+G)^{-1 / 2}$. The corresponding Mollier diagram is provided in Fig. 8(b). As a result, the enthalpy will have local maxima at $d$ and $f$ and a local minimum at point $e$. Thus, even Rayleigh processes having only one sonic point can give rise to nonclassical Mollier diagrams. As in the previous examples, the temperature necessarily has at least one local maximum if $M$ is initially sufficiently small and may or may not also have a second maximum and a minimum.

Under the general conditions on $M$ and $G$ stated earlier in this section, Mollier curves that resemble those of the well-known perfect gas theory can also be generated. These cases are those for which the Mach number is a monotone increasing function of $V$ and those for which either the local minimum in $M$ is greater than one or those for which $M=(1+G)^{-1 / 2}$ at no more than one point. Of course, flows for which the Mach number variation is monotone contain the perfect gas theory as a special case. 

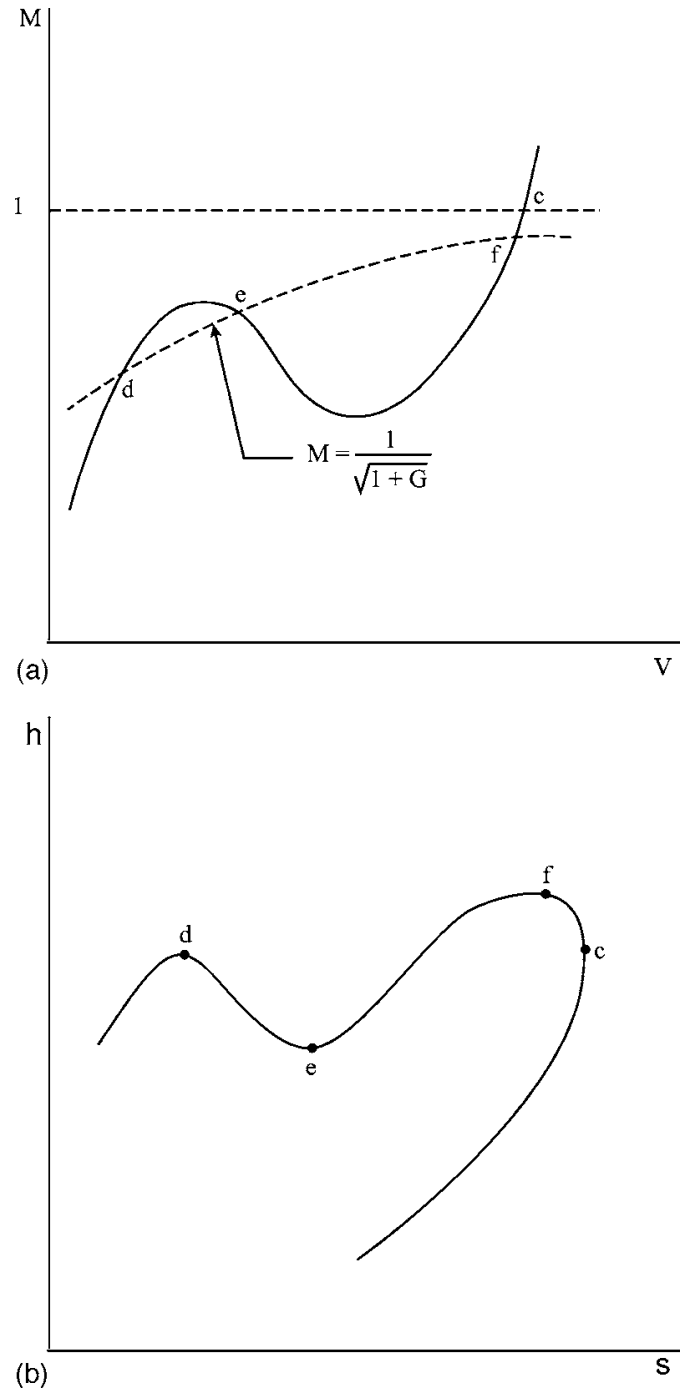

FIG. 8. $M-V$ and $h-s$ diagrams. Example of a flow having one sonic point and three extrema of $h$.

\section{EXACT SOLUTIONS FOR VAN DER WAALS GASES}

In this section we will present exact solutions for Rayleigh processes in van der Waals gases. The specific model used is given by

$$
p=\frac{R T}{V-b}-\frac{\alpha}{V^{2}},
$$

where the positive constants $b$ and $\alpha$ are related to the excluded volume and the intermolecular forces and $R$ is the gas constant. The model is completed by a specification of the ideal gas specific heat, defined as

$$
c_{v \infty}=c_{v \infty}(T)=\lim _{\rho \rightarrow 0} c_{v}(\rho, T) .
$$

The exact solutions will be presented in terms of an arbitrary temperature dependence of $c_{v^{\infty}}$.

The internal energy $e=e(V, T)$ and entropy can be determined by substituting (34) and (35) in the well-known formulas from thermodynamics, viz.,

$$
\begin{aligned}
& d e=c_{v} d T-\left(p-\left.T \frac{\partial p}{\partial T}\right|_{V}\right) d V, \\
& T d s=c_{v} d T+\left.T \frac{\partial p}{\partial T}\right|_{V} d V,
\end{aligned}
$$

which can then be integrated to yield

$$
\begin{aligned}
& e-e_{\text {ref }}=\phi(T)-\alpha\left(\frac{1}{V}-\frac{1}{V_{\text {ref }}}\right), \\
& s-s_{\text {ref }}=\psi(T)+R \ln \left(\frac{V-b}{V_{\text {ref }}-b}\right),
\end{aligned}
$$

where

$$
\phi \equiv \int_{T_{\mathrm{ref}}}^{T} c_{v^{\infty}}(T) d T, \quad \psi \equiv \int_{T_{\mathrm{ref}}}^{T} c_{v^{\infty}}(T) \frac{d T}{T},
$$

and the subscript ref denotes conditions at an arbitrary reference state. In the course of our integrations, we have recognized that the pressure given by (34) is linear in the temperature, resulting in $c_{v}(\rho, T)=c_{v \infty}(T)$ for a van der Waals gas. The enthalpy can then be computed using its definition in terms of the internal energy, i.e., $h \equiv e+p V$. The thermodynamic sound speed can be computed by noting that the general expression for the ratio of specific heats can be written as

$$
\gamma=1-T V^{2} \frac{\left(\left.\frac{\partial p}{\partial T}\right|_{V}\right)^{2}}{\left.\frac{\partial p}{\partial V}\right|_{T}}
$$

The substitution of (34), (35), and (41) in (2) and (6) then yields

$$
a=\left[\frac{R T V^{2}}{(V-b)^{2}}\left(1+\frac{R}{c_{v^{\infty}}}\right)-\frac{2 \alpha}{V}\right]^{1 / 2} .
$$

The expression for the Grüneisen parameter for the van der Waals gas can be obtained by the substitution of (34) and (35) in the last of (27) to yield

$$
G=\frac{R}{c_{v^{\infty}}} \frac{V}{V-b} .
$$

Results (38)-(40), (42), and (43) are simply those for a van der Waals gas with a nonconstant ideal gas-specific heat. Explicit formulas, parametrized by $V$, for the Rayleigh process, can now be derived by substituting (34) in the momentum, Eq. (10), to yield the expression for the temperature variation along the Rayleigh line,

$$
\begin{aligned}
T-T_{0}= & \frac{\alpha}{R}(V-b)\left(\frac{1}{V^{2}}-\frac{1}{V_{0}^{2}}\right)+\frac{\dot{m}^{2}}{R}(V-b)\left(V-V_{0}\right) \\
& -T_{0}\left(1-\frac{V-b}{V_{0}-b}\right),
\end{aligned}
$$

where the subscript 0 denotes an arbitrary initial state for the Rayleigh process. The mass flux $\dot{m}$ is determined once the initial thermodynamic state $\left(V_{0}, T_{0}\right)$ and the initial Mach 


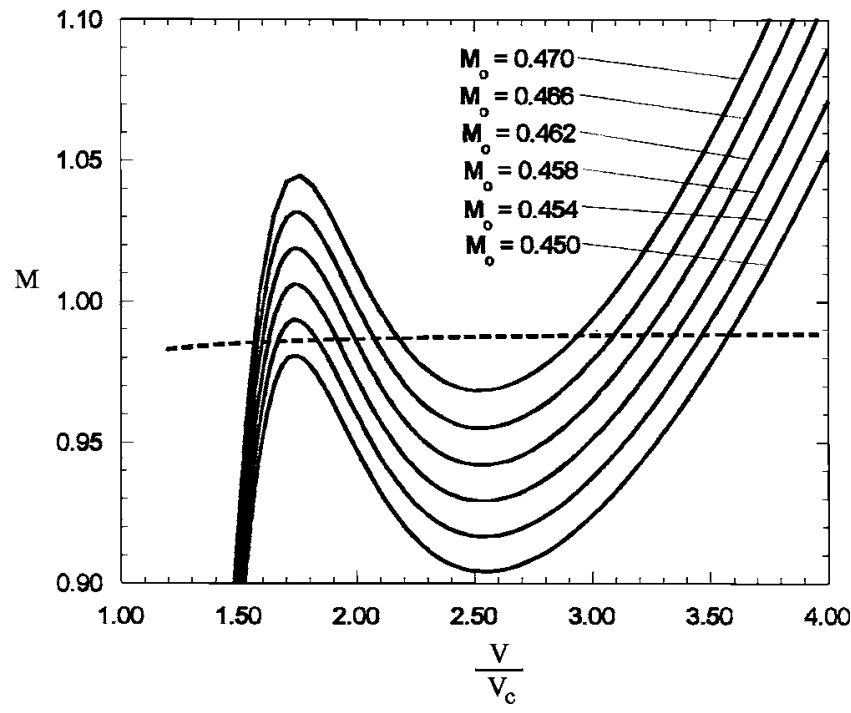

FIG. 9. Computed Mach number variation for decane $\left(\mathrm{C}_{10} \mathrm{H}_{22}\right)$ modeled as a van der Waals gas and inlet conditions $V_{0}=1.2 V_{c}, T_{0}=1.02 T_{c}$, and the indicated values of $M_{0}$. The dashed line denotes the $(1+G)^{-1 / 2}$ vs $V$ curve for each $M_{0}$.

number $M_{0}$ are specified by the following detailed form of (9):

$$
\dot{m}=\frac{M_{0} a_{0}}{V_{0}}=\frac{M a}{V} .
$$

The variation of the local Mach number, i.e., $M=M(V)$, on the Rayleigh line can be determined by substituting (42) and (44) in (45).

In order to illustrate these exact solutions and to provide a partial verification of the general deductions of Sec. III, we have applied the formulas developed here to the case of normal decane $\left(n-\mathrm{C}_{10} \mathrm{H}_{22}\right)$. The physical data for decane was taken from Reid, Prausnitz, and Poling. ${ }^{18}$ The specific heat model was taken to be a power law of the form

$$
c_{v \infty}=c_{v \infty}\left(T_{\text {ref }}\right)\left(\frac{T}{T_{\text {ref }}}\right)^{n} \text {, }
$$

where $n$ was obtained by fitting (46) to the results obtained from the more accurate polynomial fits provided by Reid, Prausnitz, and Poling. ${ }^{18}$ The reference temperature was taken to be the critical temperature $T_{c}$ of decane and the $V_{\text {ref }}$ seen in (38) and (39) was taken to be the specific volume corresponding to a pressure of one atmosphere and a temperature equal to the critical temperature, as estimated by the ideal gas law. The excluded volume and intermolecular force parameters introduced in (34) were taken to be

$$
b=\frac{R T_{c}}{8 p_{c}}, \quad \alpha=\frac{27}{64} \frac{R^{2} T_{c}^{2}}{p_{c}} .
$$

The numerical results of these evaluations are summarized in Figs. 9-12 for an initial thermodynamic state of $V_{0}=1.2 V_{c}, T_{0}=1.02 T_{c}$.

In Figs. 9 and 10 we have plotted the $M$ vs $V / V_{c}$ variation and the $\bar{h}$ vs $\bar{s}$ variation for initial Mach numbers ranging from 0.450 to 0.470 . Here

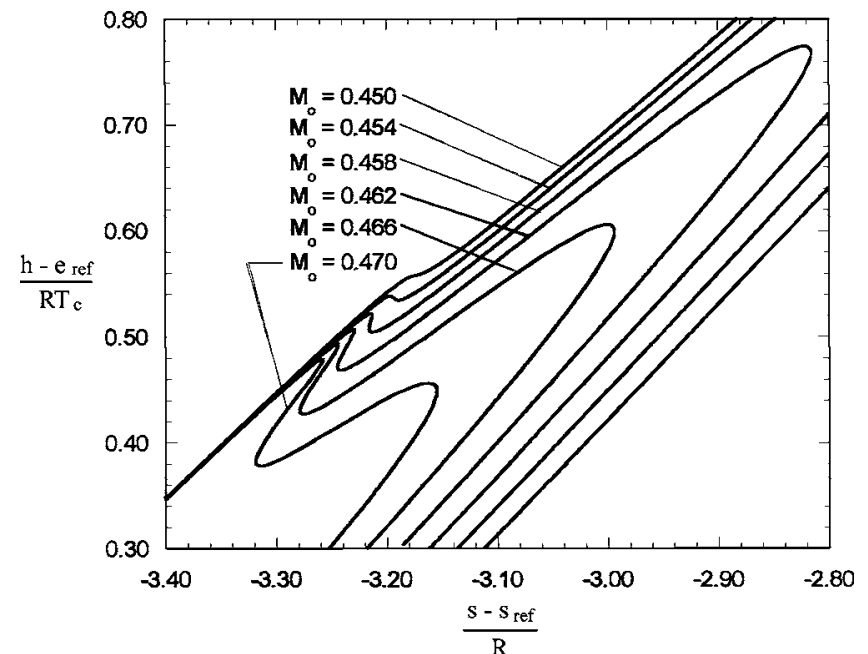

FIG. 10. Computed Mollier diagrams for decane $\left(\mathrm{C}_{10} \mathrm{H}_{22}\right)$ modeled as a van der Waals gas and inlet conditions $V_{0}=1.2 V_{c}, T_{0}=1.02 T_{c}$, and the indicated values of $M_{0}$.

$$
\bar{h} \equiv \frac{h-e_{\text {ref }}}{R T_{c}} \quad \text { and } \quad \bar{s} \equiv \frac{s-s_{\text {ref }}}{R} .
$$

In Fig. 9, we have also plotted the variation of $(1+G)^{-1 / 2}$ with $V / V_{c}$ along the Rayleigh line for each value of $M_{0}$. This variation is denoted by the dashed line. Inspection of the detailed numerical data reveals small differences in the values of $(1+G)^{1 / 2}$ along the Rayleigh line, although these differences are not noticeable on the scales used in Fig. 9.

At the lowest mass flux, i.e., for $M_{0}=0.450$, the $M$ vs $V / V_{c}$ curve intersects the $(1+G)^{-1 / 2}$ vs $V / V_{c}$ curve at only one point. As discussed in Sec. III, the Mollier diagram, i.e., the $h$ vs $s$ plot, will therefore qualitatively resemble the classical diagram with a single local maximum in both $h$ and $s$. In the interests of resolving the nonclassical features seen in Fig. 10, these maxima are not depicted in Fig. 10. However,

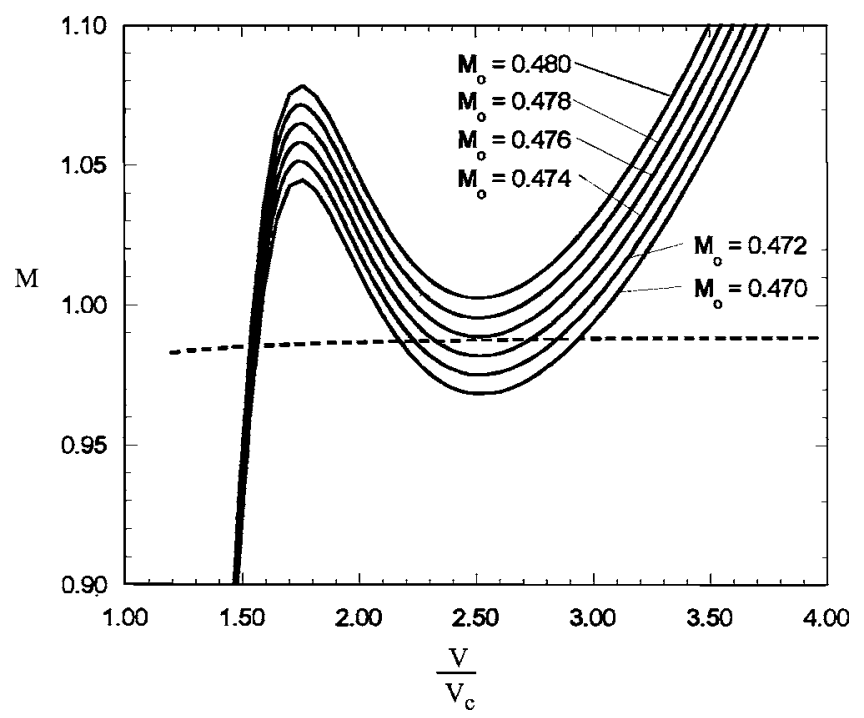

FIG. 11. Computed Mach number variation for decane $\left(\mathrm{C}_{10} \mathrm{H}_{22}\right)$ modeled as a van der Waals gas and inlet conditions $V_{0}=1.2 V_{c}, T_{0}=1.02 T_{c}$, and the indicated values of $M_{0}$. The dashed line denotes the $(1+G)^{-1 / 2}$ vs $V$ curve for each $M_{0}$. 


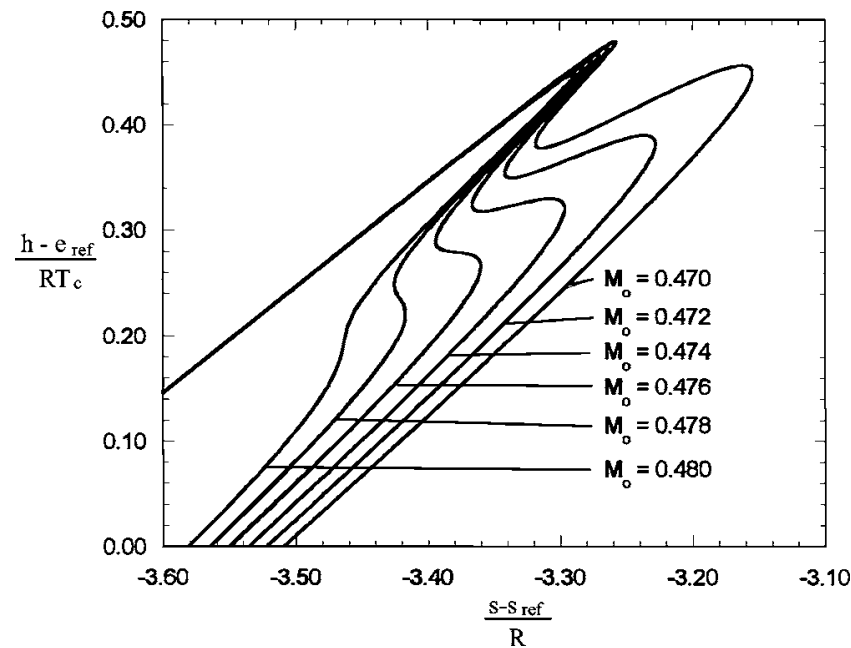

FIG. 12. Computed Mollier diagrams for decane $\left(\mathrm{C}_{10} \mathrm{H}_{22}\right)$ modeled as a van der Waals gas and inlet conditions $V_{0}=1.2 V_{c}, T_{0}=1.02 T_{c}$, and the indicated values of $M_{0}$.

an inspection of the numerical details verifies the above conclusion. As the mass flux is increased, the local maximum in the Mach number becomes large enough to result in two more intersections of the $M$ vs $V / V_{c}$ curve and the $(1+G)^{-1 / 2}$ vs $V / V_{c}$ curve. The flow having $M_{0}=0.454$ corresponds to that depicted in Fig. 8. That is, the local maximum in $M$ is large enough to result in three intersections of the $M$ vs $V / V_{c}$ and $(1+G)^{-1 / 2}$ vs $V / V_{c}$ curves, but the $M$ vs $V / V_{c}$ curve only has one sonic point. In Fig. 10 the local maximum and local minimum of $h$, corresponding to points $d$ and $e$ in Fig. $8(b)$, can be seen in the range $-3.20<\bar{s}<-3.18$. The remaining cases depicted in Figs. 9 and 10 have mass fluxes large enough to cause the local maximum in $M$ to exceed one resulting in three sonic points. For these cases, the local minimum in the Mach number remains below the $(1+G)^{-1 / 2}$ vs $V / V_{c}$ curve. As a result the curves corresponding to $M_{0}=0.458,0.462,0.466$, and 0.470 are specific examples of those represented in Fig. 7.

The Mach number variation and the Mollier diagram for initial Mach numbers ranging from $M_{0}=0.470$ to 0.480 are plotted in Figs. 11 and 12. As in Fig. 9, the dashed line in Fig. 11 denotes the $(1+G)^{-1 / 2}$ vs $V / V_{c}$ for each value of $M_{0}$. As the initial Mach number is increased from 0.470, the subsonic portion of the flow in the vicinity of the local minimum of $M$ becomes smaller, ultimately resulting in the value of the low-pressure entropy maximum (corresponding to point $c$ in Fig. 7) becoming smaller than the value of the highpressure entropy maximum. However, the most significant qualitative change occurs when the local minimum in $M$ becomes large enough to pass above the $(1+G)^{-1 / 2}$ vs $V / V_{c}$ curve; in the case considered here, this transition occurs at a $M_{0}$ slightly less than 0.476 . When the minimum value of $M$ lies above the $(1+G)^{-1 / 2}$ vs $V / V_{c}$ curve and below one, the flow has three sonic points, two maxima in entropy, one minimum in entropy, but only a single local maximum in the enthalpy. The flow is of the type depicted in Fig. 6. In Figs. 11 and 12 the curves corresponding to $M_{0}=0.476$ and 0.478 are of this type. As the mass flux is further increased, the local minimum in $M$ exceeds one, resulting in only one sonic point, one local maximum in $s$ and $h$, and therefore a flow that is qualitatively similar to classical Rayleigh flows. An example of such a quasiclassical flow is that corresponding to $M_{0}=0.480$ in Figs. 11 and 12.

\section{SHOCKED AND UNSHOCKED FLOWS}

In this section we consider flows that are either strictly heated $\left(Q^{\prime}>0\right)$ or strictly cooled $\left(Q^{\prime}<0\right)$. Here the primes denote differentiation with respect to $x$, which is a coordinate aligned with the flow direction and is positive in the direction of flow. In the following discussion we will provide a comprehensive description of the shocked and unshocked flows possible under strict heating or cooling.

We will confine our attention to flows corresponding to Rayleigh lines of the type depicted in Fig. 4. In flows having $\Gamma>0$ at every point, the possible solutions are qualitatively the same as those predicted in the perfect gas theory. In particular, when $\Gamma>0$ at every point, a heated flow with a fixed initial thermodynamic state and fixed initial Mach number will always choke at the position corresponding to the maximum value of $Q$. Cooled flows will not choke regardless of the amount of heat extracted. In cases where $\Gamma<0$ at every point in the Rayleigh flow, we simply need to reverse the roles of heating and cooling. That is, when $\Gamma<0$ at every point in a heated flow, no choking can occur regardless of the amount of heat added. Cooled $\Gamma<0$ flows will be seen to choke at a point corresponding to a local minimum in $Q$. The conclusions regarding both $\Gamma>0$ and $\Gamma<0$ flows can be obtained straightforwardly by the arguments provided in the remainder of this section. As a result, we will not provide a detailed discussion here.

We now consider flows having Rayleigh lines that pass through the $\Gamma<0$ region depicted in Fig. 1; the corresponding $p-V$ diagrams are sketched in Fig. 4 . In each case discussed next, the specific Rayleigh line will be regarded as fixed. As a result, the variation of $Q$ with $V$ will be known. As pointed out in Sec. III, the $Q$ vs $V$ curves will appear qualitatively the same as the $s$ vs $V$ curves sketched in Fig. 5; typical $Q$ vs $V$ curves are sketched in Fig. 13. Because we have restricted our attention to strictly heated or strictly cooled flows, i.e., monotone variations of $Q$ with $x$, the form of the $V$ vs $x$ curves can be easily deduced from the $V$ vs $Q$ curves.

Examples of the variation of $V$ for heated and cooled flows are sketched in Fig. 14. For the heated flow, the initial $(x=0)$ state can be regarded as either being so far to the left of point $a$ in Fig. 4 or 13 or so far to the right of point $c$ that $Q(0)<Q_{\min }$, where $Q_{\min }$ is the local minimum of $Q$ corresponding to point $b$ in Figs. 4, 5, and 13. As in the classical perfect gas theory, flow heating with fixed initial conditions ultimately always results in a choked flow. However, in the case of the BZT fluid depicted in Fig. 14(a), subsonic initial conditions result in choking at point $a$ whereas supersonic initial conditions result in choking at the low-pressure sonic point at $c$. The $V$ vs $x$ curves for other initial conditions have been sketched in the remainder of this section. In each case, 

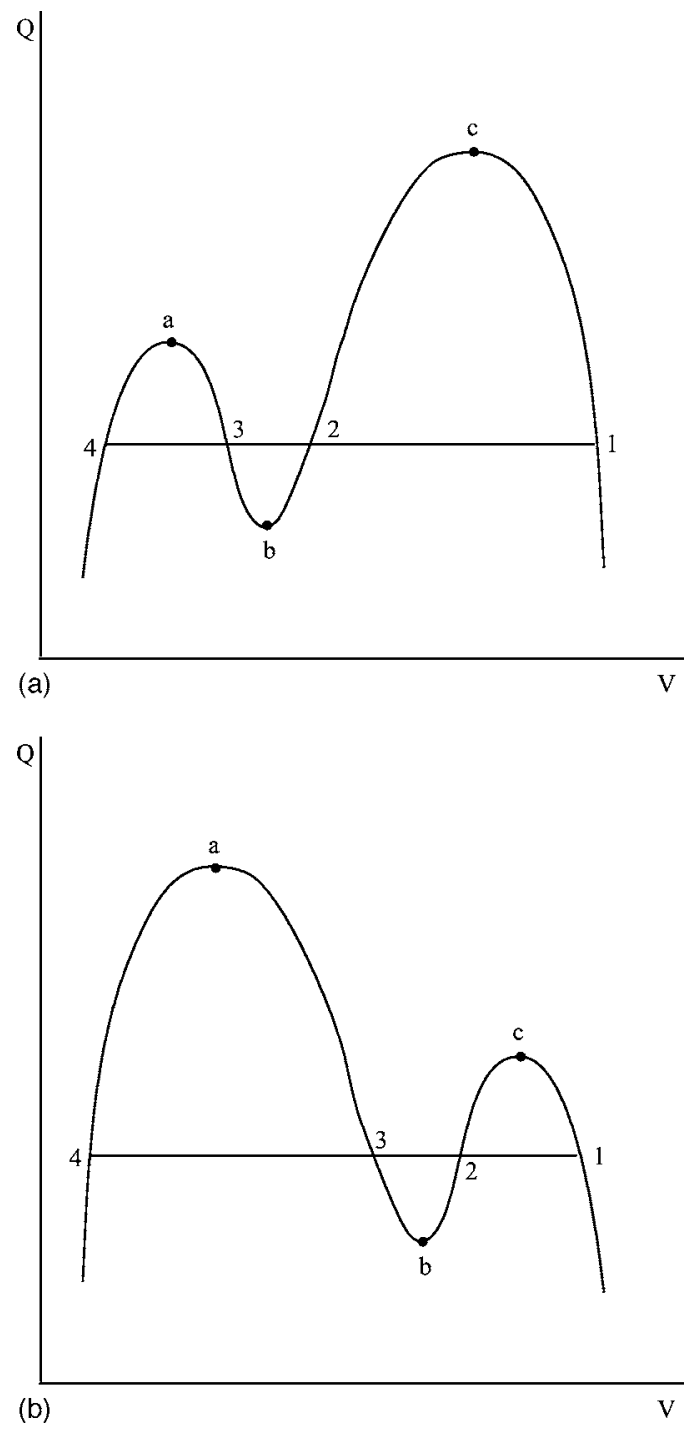

FIG. 13. Variation of $Q$ along a Rayleigh line. The points 1, 2, 3, 4 represent solutions to the Rankine-Hugoniot conditions (12)-(14). (a) $Q_{c}>Q_{a}>Q_{b}$; (b) $Q_{a}>Q_{c}>Q_{b}$.

unshocked flows with fixed initial conditions will always choke at one of the maxima of $Q$.

An example of an unshocked cooled flow is sketched in Fig. 14(b). The two possible initial states are in the vicinity of the largest local maximum of $Q$. If the initial Mach number is less than one, i.e., if the flow starts at point 01, the flow may be cooled indefinitely. On the other hand, flow initiated on the supersonic branch must ultimately choke at the local minimum of $Q$; this point corresponds to point $b$ in Figs. 4, 5 , and 13. This result contrasts with the classical theory in which cooled flows do not choke. The $V$ vs $x$ curves for the remaining cooled flow cases have been sketched in Figs. 21 and 23, later. Unless the initial conditions are chosen to be so far to the left of point $a$ in Figs. 4 and 13 or so far to the right at point $c$ that $Q(0)<Q_{\min }$, where $Q_{\min }$ is the local minimum of $Q$ corresponding to point $b$ in Figs. 4 and 13, initial conditions leading to choking at $Q_{\min }\left(=Q_{b}\right)$ will always exist.

We now consider shocked flows. As pointed out in Sec. II, shock waves will correspond to the condition $Q=$ const. As a result, shocks will appear as horizontal lines in a $Q$ vs $V$
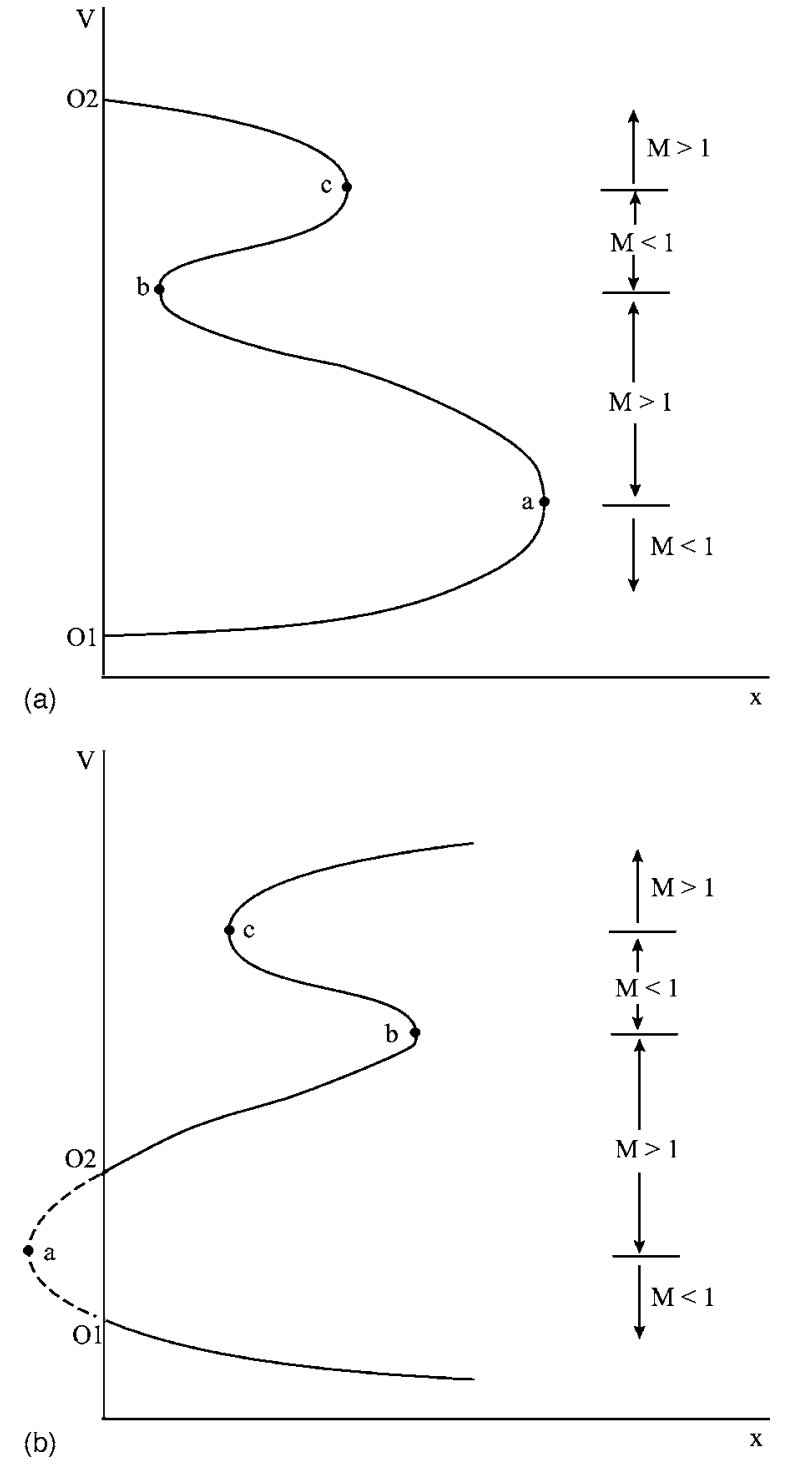

FIG. 14. Sample $V$ vs $x$ variations for unshocked flows. (a) Strictly heated flow; (b) strictly cooled flow.

plot; the line 1-2-3-4 sketched in Fig 13 is an example of such a $Q=$ const line. Each intersection of the $Q=$ const line with the $Q$ vs $V$ curve represents a state satisfying (12)-(14). Because there are four intersections of the $Q=$ const and $Q$ vs $V$ curves, points 1, 2, 3, 4 in Fig. 13 correspond to the intersection points 1, 2, 3, 4 seen in Fig. 3, respectively.

Because $Q=Q(x)$, we obtain the intuitively obvious result that the shock occurs at a fixed value of $x$ so that it appears as a discontinuity in the $V-x$ plane. Because we have restricted our attention to cases having $Q^{\prime} \neq 0$, the shock position is unique.

Our selection of admissible shock waves, i.e., physically realizable discontinuities, will necessarily require the use of the shock admissibility condition stated in Sec. II. If we combine our statement of the admissibility condition with the fact that the slopes of both the Rayleigh line and the shock adiabat are negative in the $p-V$ plane, we can conclude that admissible shocks necessarily correspond to neighboring intersections of the Rayleigh line and the shock adiabat. The admissible shocks in the $Q-V$ planes must also correspond to 


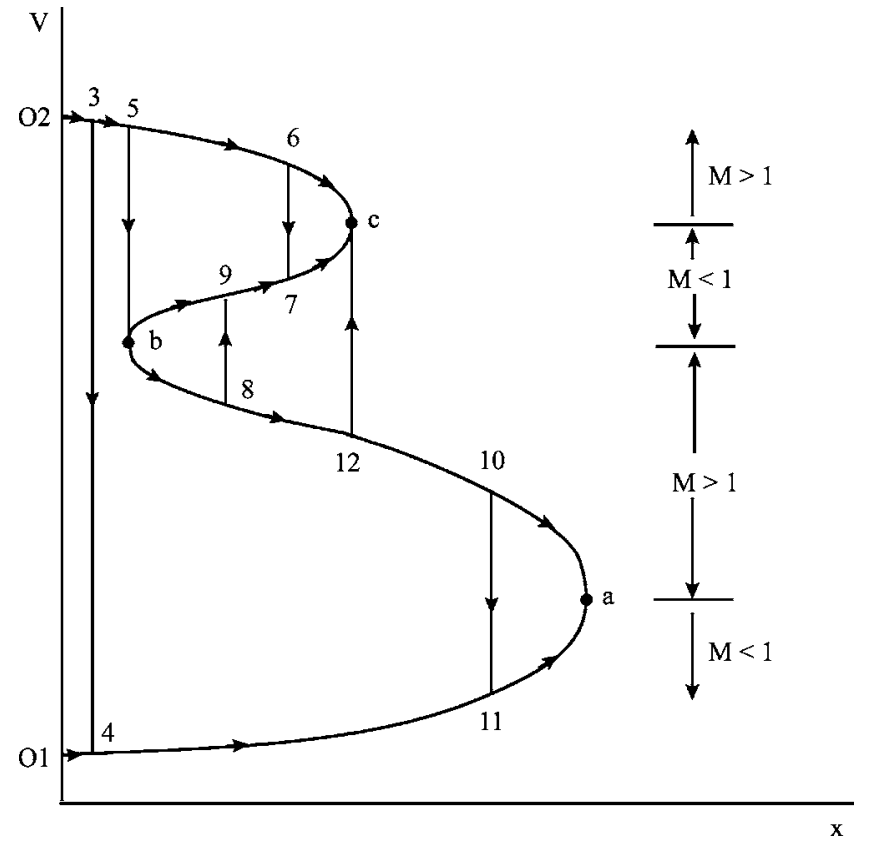

FIG. 15. Shocked strictly heated flows for $Q_{a}>Q_{c}>Q_{b}>Q(0)$.

neighboring intersections of the $Q=$ const and $Q$ vs $V$ lines. In the $V$-x plane, e.g., those depicted in Fig. 14, admissible shocks will correspond to vertical lines that do not intersect the $V$ vs $x$ curve between the upstream and downstream states. In order to ascertain whether the admissible shock is a compression or expansion shock we need only employ the necessary speed-ordering condition (20). That is, the direction of the jump will necessarily take the flow from either supersonic or sonic conditions to either subsonic or sonic conditions.

Although we will not use it explicitly in the following analysis, it can be shown that the admissibility condition of Sec. II can be restated in the following manner for Rayleigh flows: An admissible compression (expansion) shock is one for which the $Q$ vs $V$ curve lies entirely above (below) the $Q=$ const line between its upstream and downstream states. This statement holds for all $Q$ vs $x$ variations, i.e., the $Q$ vs $x$ variation may or may not be monotone.

We now describe all shocked configurations possible for strictly heated or strictly cooled Rayleigh flows having a Rayleigh line that passes through the $\Gamma<0$ region depicted in Fig. 1. We first consider a strictly heated flow where the $Q$ vs $V$ plot is of the type depicted in Fig. 13(b), i.e., one for which $Q_{a}>Q_{c}>Q_{b}>Q(0)$. The $V$ vs $x$ variation is resketched in Fig. 15 along with selected admissible shock waves.

In our discussion of unshocked flows, we noted that a flow having a subsonic initial state, say $V=V_{01}$, will choke at the sonic point $a$. Even if we account for shock waves, this conclusion remains valid for the case considered here. However, if the initial state corresponds to a supersonic state, e.g., that initiated at 02 , the flow may or may not choke at point $c$. Choked flows reminiscent of those found in the classical theory include those of the type 02-3-4- $a$ and 02-6-7-c. In each case, a compression shock, 3-4 or 6-7, takes the flow to

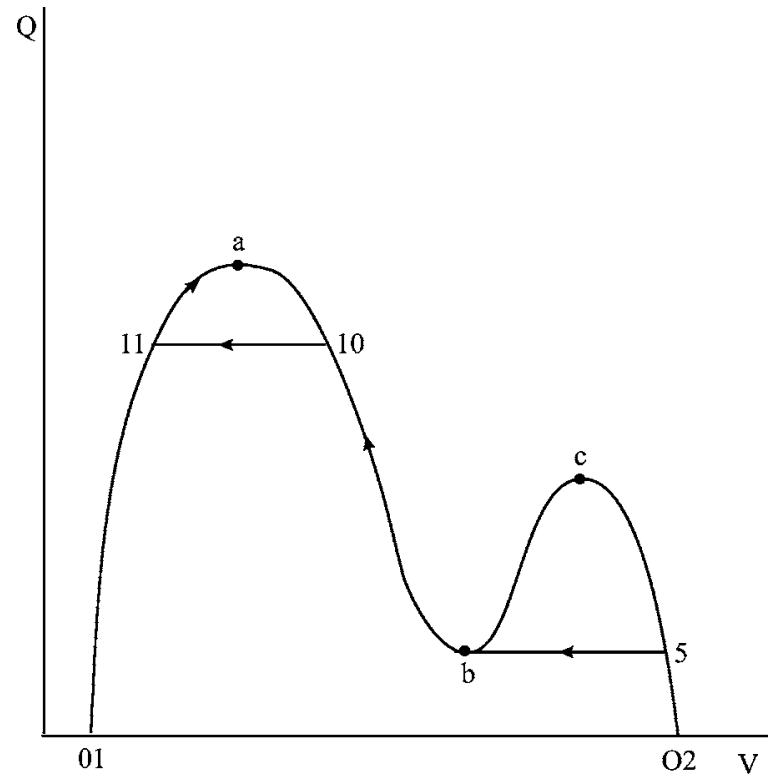

FIG. 16. Variation of $Q$ for the split-shock case of Fig. 15.

a subsonic branch after which the flow accelerates in a shock-free manner to one of the sonic points.

A series of nonclassical flows contain the sonic compression shock $5-b$; this shock is analogous to the sonic shock 1-5 depicted in Fig. 3. From the sonic point b, the flow can either expand subsonically to the sonic point $\mathrm{c}$ or may compress supersonically along the branch $b-a$. In cases where the flow follows the supersonic branch $b-a$, a second shock may be formed. If the second shock is of the expansion type, e.g., $8-9$, the flow will ultimately choke at the low-pressure sonic point $c$. The expansion shock of maximum strength is the downstream sonic shock, depicted as 12-c in Fig. 15. If the shock is a compression shock of the type 10-11, the flow will ultimately choke at the high-pressure sonic point $a$. It is of interest to note that there are a total of three flows for which the flow chokes without becoming subsonic. These are the shock-free flow 02-c analogous to that of the classical theory and the shocked flows 02-5-b-a and 02-5-b-12-c.

The compression shocks seen in flows of the type 02-5$b-10-11$ will be referred to as split shocks or as representing shock splitting. Similar split-shock configurations have also been predicted in nozzle flows ${ }^{5,6}$ and Fanno flows. ${ }^{7}$ Shock splitting has also been described in the context of the existence and disintegration of compression shocks by Cramer ${ }^{12}$ and Menikoff and Plohr. ${ }^{16}$ In order to provide an example of the representation of shocked Rayleigh flows in the $p-V$ plane we have sketched the $Q$ vs $V$ curve and the Rayleigh line and shock adiabats corresponding to the split-shock flow 02-5- $b-10-11-a$ in Figs. 16 and 17. In Fig. 17, the Rayleigh line is the straight line $02-5-c-b-10-a-11$ and is regarded as fixed. The shock adiabat corresponding to the shock 5- $b$ seen in Figs. 15 and 16 is the unique adiabat that is tangent to the Rayleigh line at the $\Gamma<0$ sonic point $b$; we denote this adiabat as $H_{5}$. The flow therefore proceeds from 02 to the intersection 5 and then undergoes a compression shock from 5 to $b$. The flow then proceeds from $b$ to 10 along the Rayleigh line, as indicated in Figs. 16 and 17. Because $b$ corresponds 


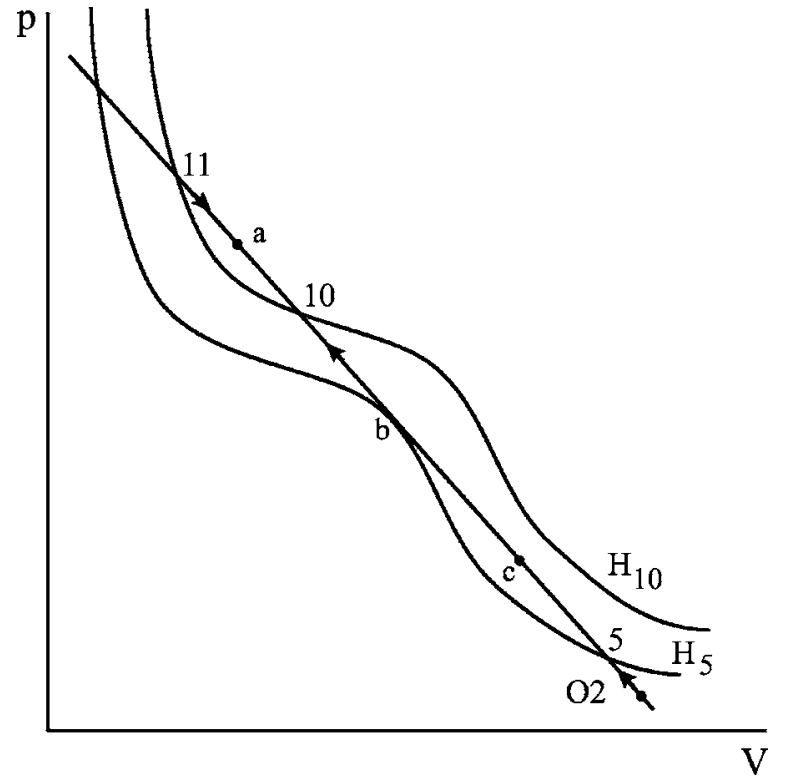

FIG. 17. Rayleigh line and shock adiabats for the split-shock case of Fig. 15.

to a local minimum in $Q, Q_{10}>Q_{b}$, and, as discussed in Sec. II, the shock adiabat associated with 10-11, denoted by $H_{10}$, lies above $H_{5}$ in the $p-V$ plane. After passing through the compression shock 10-11, the flow then expands along the Rayleigh line from 11 to $a$ as sketched in Figs. 16 and 17.

Because the compression shock 10-11 was sketched having $x_{10-11}>x_{c}$ in Fig. 15, and therefore $Q_{10-11}>Q_{c}$ in Fig. 16, there can be no more than two intersections of the adiabat $H_{10}$ and the Rayleigh line. Thus, $H_{10}$ has been sketched with only two intersections in Fig. 17. However, if a shock of the type 10-11 occurs sufficiently close to point $b$, i.e., such that $Q_{b}<Q_{10-11}<Q_{c}$, the adiabat $H_{10}$ should be sketched with four intersections between it and the Rayleigh line.

We now consider strictly heated flows for which $Q_{c}>Q_{a}>Q_{b}>Q(0)$. In this case, most of the flows are qualitatively the same as those described for the $Q_{a}>Q_{c}$ $>Q_{b}>Q(0)$ case. The primary differences between these two cases are depicted in Fig. 18. The first case of interest is the initially subsonic case that begins at 01 and then expands subsonically to the sonic point $a$. Because $Q_{c}>Q_{a}$

Therefore $x_{c}>x_{a}$ in the $V-x$ plane; the solution can be continued downstream through the upstream sonic expansion shock $a-8$. After passing through this expansion shock, the flow can proceed subsonically to the low-pressure sonic point $c$. Thus, the main new feature is the occurrence of the sonic shock $a-8$ and the fact that the mass flow is ultimately limited by choking at $c$ rather than $a$.

A second flow that can occur when $Q_{c}>Q_{a}>Q_{b}$ $>Q(0)$ is that of the type 02-3-b-4-5-a-8-c. This case contains the shock splitting seen in Fig. 15, but a third shock, namely, the sonic expansion shock $a-8$, can also occur.

We now consider the strictly heated case where the initial state is such that $Q_{a}>Q_{c}>Q(0)>Q_{b}$. The resultant $V$ vs $x$ curve is sketched in Fig. 19. The dashed line in the $x<0$ region is not a possible solution but is simply included for comparison to the previous cases. The simplest way to view

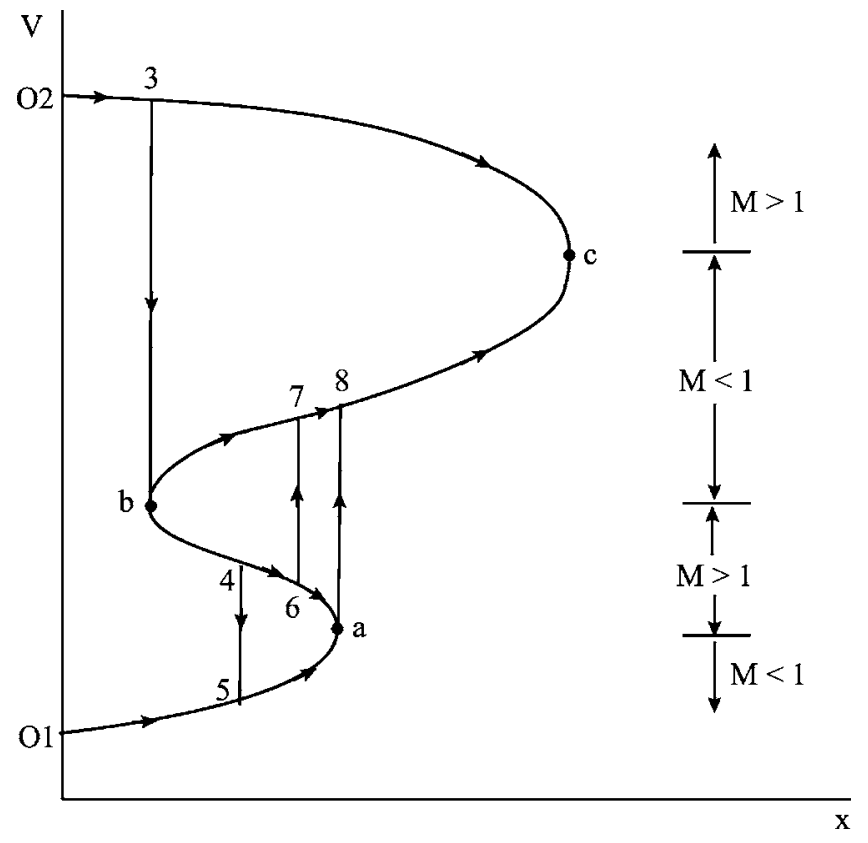

FIG. 18. Shocked strictly heated flow for $Q_{c}>Q_{a}>Q_{b}>Q(0)$.

these flows is as reinitializations (in $x$ ) of those seen in the $Q_{a}>Q_{c}>Q_{b}>Q(0)$ case. As a result, we will minimize our detailed discussion of this case. We note that flows initiated at $01,03,04$ will have the same qualitiative features as those of the classical theory. However, those initiated at the supersonic point 02 can either follow the classical paths $02-a$, 02-10-11- $a$ or a nonclassical path involving nonsonic expansion shocks of the type 7-8 or the downstream sonic shock 9-c. We note that no more than one shock can appear in any strictly heated flow having $Q_{a}>Q_{c}>Q(0)>Q_{b}$.

The next case to be considered is that having $Q_{c}>Q_{a}$ $>Q(0)>Q_{b}$, i.e., it is essentially the same as that depicted Fig. 19 except that the local maximum of $Q$ at $c$ is greater than the local maximum of $Q$ at $a$. A sketch of the $V$ vs $x$

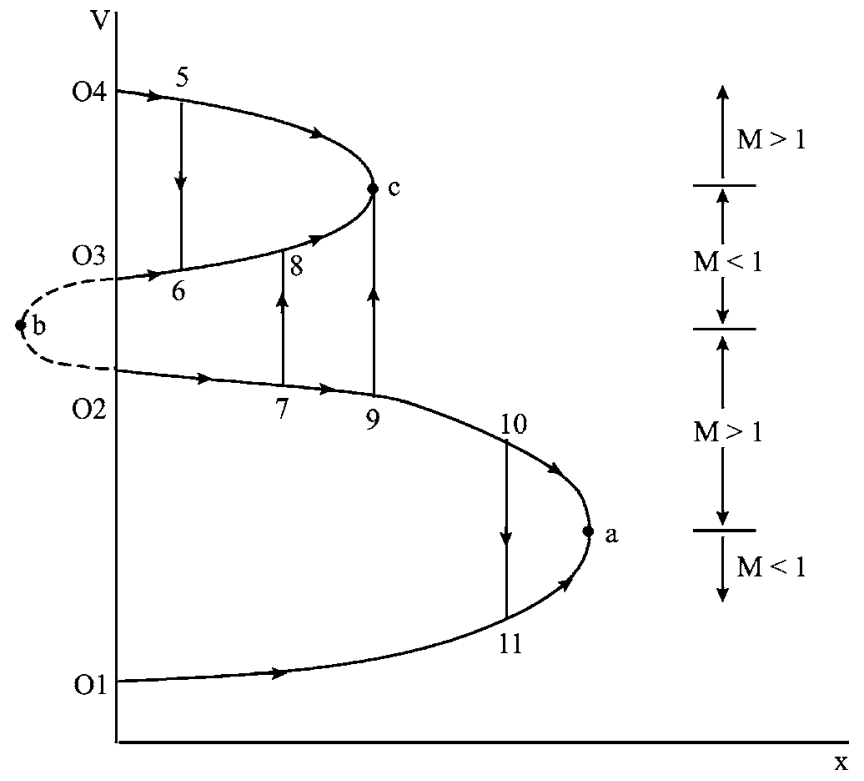

FIG. 19. Shocked strictly heated flows for $Q_{a}>Q_{c}>Q(0)>Q_{b}$. 


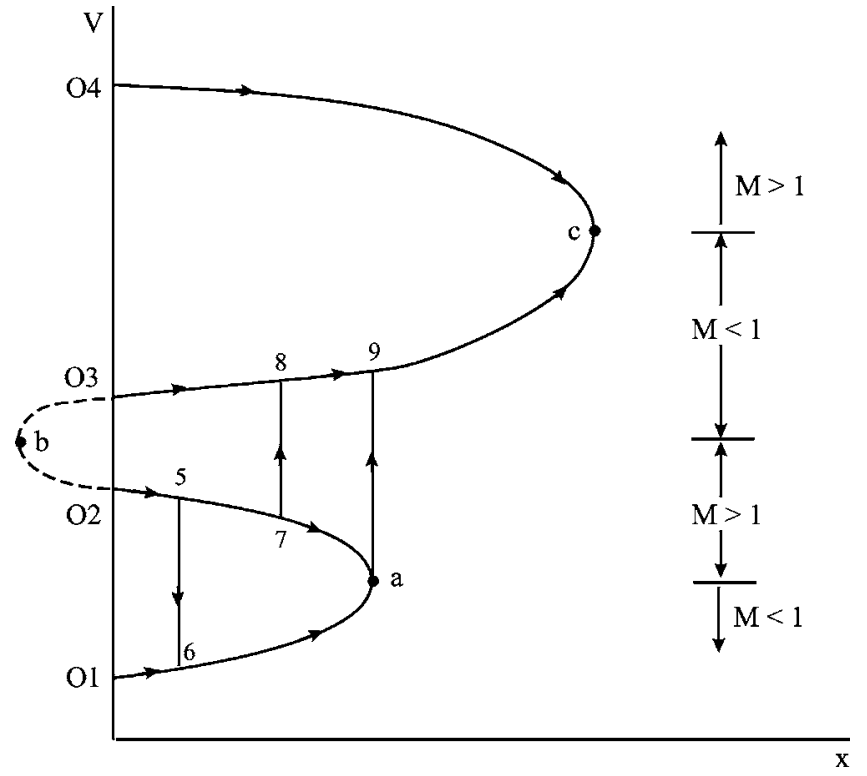

FIG. 20. Shocked strictly heated flows for $Q_{c}>Q_{a}>Q(0)>Q_{b}$.

curve is provided in Fig. 20 and again is seen to be just a reinitialization of the flow depicted in Fig. 18. As in Fig. 18, we only include details for flows that are fundamentally different than those of the case depicted in Fig. 19. The primary new feature is the possibility of the upstream sonic shock $a-9$, which is seen to be of the type as $a-8$ seen in Fig. 18 . Thus, a flow initiated at the subsonic point 01 can shock to the upper branch and ultimately choke at point $c$ instead of point $a$.

The last two subcases that involve strictly heated flows correspond to $Q_{c}>Q(0)>Q_{a}>Q_{b}$ and $Q_{a}>Q(0)>Q_{c}$ $>Q_{b}$. In each case the $Q$ vs $V$ and $V$ vs $x$ variations and qualitatively the same as those of the classical perfect gas theory, and we will not provide a detailed discussion here.

We now consider strictly cooled flows, i.e., those having $Q^{\prime}<0$. As pointed out at the beginning of this section, the $V$ vs $x$ curves for the $Q^{\prime}<0$ flows will be the same as the $Q^{\prime}$ $>0$ curves but reflected about the $V$ axis. The first subcases to be considered are the analogs of those depicted in Figs. 15 and 18, i.e., $Q_{a}, Q_{c}>Q_{b}>Q(0)$. The resultant $V$ vs $x$ curves are qualitatively the same as those of the classical perfect gas or more general $\Gamma>0$ theory described earlier in this section. The second set of subcases is that for which $Q_{a}, Q_{c}>Q(0)$ $>Q_{b}$; the resultant $V$ vs $x$ curves have been sketched in Fig. 21. As in previous examples the curves shown as dashed lines do not represent possible flows. Rather, they are provided for reference only.

Shock-free flows initiated at the subsonic point 01 and the supersonic point 04 and the shocked flow 04-12-13 are qualitatively the same as those of the classical $Q^{\prime}<0$ theory. If, in a flow that is initiated at 04 , the shock wave occurs at a point $x<x_{b}$, then the flow will either choke at $b$ or will generate a split-shock configuration of the type 04-9-10$b-11$. The two unshocked flows $02-b$ and $03-b$ and the shocked flow 02-5-6- $b$ are qualitatively the same as those described for $\Gamma<0$ flows. As sketched in Fig. 21, flows that are initiated at the subsonic point 03 or the supersonic point

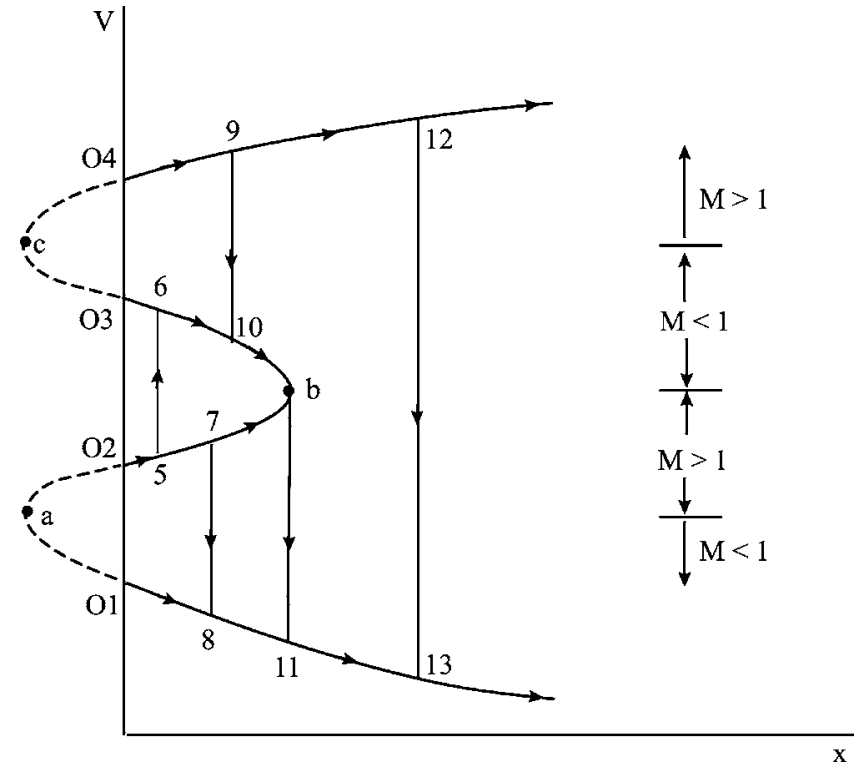

FIG. 21. Shocked strictly cooled flows for $Q_{a}, Q_{c}>Q(0)>Q_{b}$.

02 can be shocked to the lower subsonic branch, which intersects the $x=0$ axis at point 01 . Because the flow initiated at 03 is subsonic, a shock can be generated only at the sonic point $b$. On the other hand, nonsonic compression shocks of the type 7-8 as well as the upstream sonic shock $b-11$ are possible for the flow initiated at the supersonic point 02 .

The next strictly cooled case to be considered is that having $Q_{a}>Q(0)>Q_{c}>Q_{b}$. The $V$ vs $x$ curve is identical to that sketched in Fig. 14 and is resketched in Fig. 22 along with a few representative shock waves. If the flow is initiated at 01 , the possible flows resemble the classical case in that they can only compress subsonically to lower and lower specific volumes. On the other hand, if the flow is initiated at the supersonic point 02 , the flow can either choke at the $\Gamma<0$ sonic point $b$ by following the shock-free path $02-b$ or by following shocked evolutions of the type $02-3-c-b$,

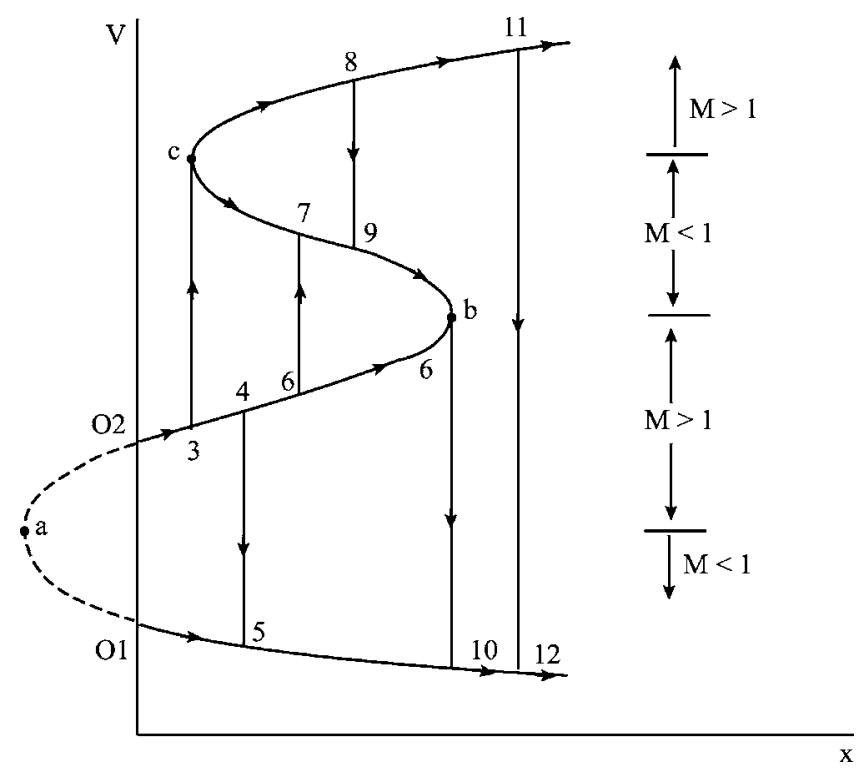

FIG. 22. Shocked strictly cooled flows for $Q_{a}>Q(0)>Q_{c}>Q_{b}$. 


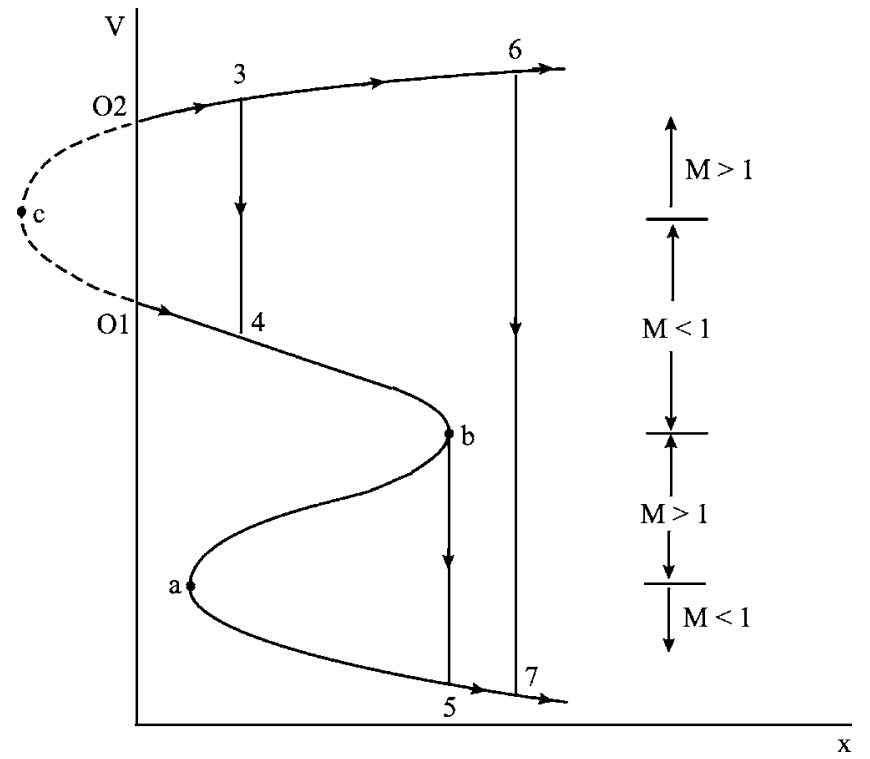

FIG. 23. Shocked strictly cooled flows for $Q_{c}>Q(0)>Q_{a}>Q_{b}$.

02-6-7-b, or 02-3-c-8-9-b. Unlike the situation for the unshocked flows, choking at $b$ can be avoided and shocked solutions include those which proceed along the upper supersonic branch, e.g., 02-3-c-8-11, and those that ultimately proceed along the lower subsonic branch. Examples of the latter include the split-shock configuration, i.e., 02-3-c-8-9-b-10. We note that, in this case, the split shock is only possible if the first compression shock is preceded by the downstream sonic expansion shock 3-c.

The last nonclassical case to be considered is that where $Q_{c}>Q(0)>Q_{a}>Q_{b}$ and is sketched in Fig. 23. This case differs from all previous nonclassical cases in that no expansion shocks are possible. As indicated in this sketch, no more than two shocks can occur, and this is only possible if the split-shock configuration $02-3-4-b-5$ is realized.

\section{SUMMARY}

The present study has provided an analysis of Rayleigh flows of general single-phase fluids. The largest differences with the classical perfect gas theory occur when the fluid is of the BZT type and the Rayleigh line passes through a region of negative nonlinearity similar to that depicted in Fig. 1. In such flows, as many as three sonic points and three extrema of the entropy and heat supply $Q$ can occur on a Rayleigh line. These results are in marked contrast with the classical theory of perfect gases and low-pressure liquids in which only one sonic point and local maximum in entropy and $Q$ can occur. Although much of the analysis found in Sec. III is valid under the standard conditions stated in Sec. II, the Mollier diagrams, i.e., the $h-s$ curves seen in Figs. 6-8 are constructed subject to mild additional assumptions. Under these conditions, as many as three extrema of the enthalpy can occur on a Rayleigh line. From the general result (31), it is seen that these extrema occur whenever $M=(1+G)^{-1 / 2}$.

In Sec. IV we have also provided exact solutions for Rayleigh flows for the special case of a van der Waals gas. We have used these exact solutions and well-established physical data for normal decane to illustrate the general theory described in Sec. III. Each of the nonclassical cases described in Sec. III, as well as cases having qualitatively the same behavior as the classical theory, can be generated by simply varying the mass flux at a fixed entrance thermodynamic state.

The general theory of flow variations has also been provided for strictly heated and strictly cooled flows. Both shocked and unshocked flows were examined and were found to differ significantly from those of the classical theory when BZT fluids were considered. These differences include flow choking in unshocked strictly cooled flows, the existence of as many as three shock waves in both cooled and heated flows, and shock splitting analogous to that reported by Cramer and Fry, ${ }^{5}$ Kluwick, ${ }^{6}$ and Cramer, Monaco, and Fabeny ${ }^{7}$ for nozzle and Fanno flows.

${ }^{1}$ G. H. Schnerr and P. Leidner, "Diabatic supersonic flows of dense gases," Phys. Fluids A 3, 2445 (1991).

${ }^{2}$ M. S. Cramer, "Nonclassical dynamics of classical gases," Virginia Polytechnic Institute and State University Report VPI-E-89-20, Blacksburg, VA, 1989.

${ }^{3} \mathrm{D}$. Chandrasekar and $\mathrm{Ph}$. Prasad, "Transonic flow of a fluid with positive and negative nonlinearity through a nozzle," Phys. Fluids A 3, 427 (1991).

${ }^{4}$ M. S. Cramer and L. M. Best, "Steady isentropic flows of dense gases," Phys. Fluids A 3, 219 (1991).

${ }^{5}$ M. S. Cramer and R. N. Fry, "Nozzle flows of dense gases," Phys. Fluids A 5, 1246 (1993).

${ }^{6}$ A. Kluwick, "Transonic nozzle flow of dense gases," J. Fluid Mech. 247, 661 (1993).

${ }^{7}$ M. S. Cramer, J. F. Monaco, and B. M. Fabeny, "Fanno processes in dense gases," Phys. Fluids 6, 674 (1994).

${ }^{8} \mathrm{H}$. A. Bethe, "The theory of shock waves for an arbitrary equation of state," Office of Scientific Research and Development Report No. 545, 1942.

${ }^{9}$ Ya. B. Zel'dovich, "On the possibility of rarefraction shock waves," Zh. Eksp. Teor. Fiz. 4, 363 (1946).

${ }^{10} \mathrm{~K}$. Lambrakis and P. A. Thompson, "Existence of real fluids with a negative fundamental derivative $\Gamma$," Phys. Fluids 15, 933 (1972).

${ }^{11}$ P. A. Thompson and K. Lambrakis, "Negative shock waves," J. Fluid Mech. 60, 187 (1973).

${ }^{12}$ M. S. Cramer, "Shock splitting in single-phase gases," J. Fluid Mech. 199, 281 (1989)

${ }^{13}$ M. S. Cramer, "Nonclassical dynamics of classical gases," in Nonlinear Waves in Real Fluids, edited by A. Kluwick (Springer, New York, 1991), p. 91.

${ }^{14}$ P. D. Lax, "Shock waves and entropy," in Contributions to Nonlinear Functional Analysis, edited by E. H. Zarantonello (Academic, New York, 1971).

${ }^{15} \mathrm{O}$. A. Oleinik, "Uniqueness and stability of the generalized solution of the Cauchy problem for a quasi-linear equation,” Am. Math. Soc. Transl. 33, 285 (1963).

${ }^{16}$ R. Menikoff and B. Plohr, "Riemann problem for fluid flow of real materials," Rev. Mod. Phys. 61, 75 (1989).

${ }^{17}$ V. Arp, J. M. Persichetti, and C. Guo-bang, "The Grüneisen parameter in fluids," J. Fluids Eng. 106, 193 (1984).

${ }^{18}$ R. C. Reid, J. M. Prausnitz, and B. E. Poling, The Properties of Gases and Liquids, 4th ed. (Wiley, New York, 1987). 